\title{
BOSQUE DE LA LLANURA ALUVIAL DEL PERÚ: ECOSISTEMAS, HABITANTES Y USO DE LOS RECURSOS
}

\author{
Lars Peter Kvist ${ }^{1}$ y Gustav Nebel ${ }^{1}$
}

\section{RESUMEN}

La Amazonía baja del Perú está muy poco poblada y mantiene una densa cobertura de bosques, en donde existen pocas carreteras y los ríos constituyen la mayor infraestructura disponible. Más del 12\% del área se compone de llanuras aluviales inundadas por los grandes ríos, llanuras que debido a su fácil acceso y a que presentan una fertilidad relativa, son fuentes de abastecimiento de la mayor parte de los recursos naturales que son extraídos en la región, y que permiten el sostenimiento de la mayoría de las poblaciones rurales. El área más grande de tierra inundada anualmente, se extiende a más de $60000 \mathrm{~km}^{2}$ y rodea las zonas bajas de los ríos Ucayali y Marañón arriba de su confluencia con el mismo Amazonas, abarcando casi el 100\% de los $20600 \mathrm{~km}^{2}$ de la gran Reserva Nacional Pacaya-Samiria. El área completa se encuentra en dinámica constante debido a la erosión y la deposición ocasionada por los dos ríos principales, y también por los ríos más pequeños que arrastran pocos sedimentos y menos nutrientes. La vegetación constituye un complejo mosaico de hábitat definido por la combinación de características hidrológicas, físicas, químicas y biológicas. Se han descrito y clasificado 16 hábitat, incluyendo 12 formaciones de bosques. La mayoría de los habitantes de la llanura aluvial ha perdido su identidad nativa, pero, mayormente éstos descienden de amerindios, y no de inmigraciones recientes, y preservan mucho conocimiento sobre el entorno de la llanura aluvial, sus habitantes y sus usos. Se describen sus actividades económicas realizadas dentro de las formaciones de bosque. Combinan la agricultura, la pesca, la caza y la extracción de otros productos del bosque, y ponen a la venta cantidades crecientes de estos productos. El aumento de la población y el incremento de la necesidad de ingresos monetarios, así como de las intervenciones económicas externas, ponen en peligro de extinción a plantas, animales y peces de la llanura aluvial que son económicamente importantes. Inclusive, esto es lo que ya está ocurriendo dentro de la Reserva Nacional Pacaya-Samiria, tornándose urgente el desarrollo e implementación de sistemas sólidos de manejo en las zonas marginales de la Reserva, y en cualquier parte de la llanura aluvial del Perú.

1 Royal Veterinary and Agricultural University, Departament of Economics and Natural Resources, Unit of Forestry, Rolighedavaj 23, 1958 Frederikaberg C. Denmark. 
Palabras claves: Pantanos, Amazonas, recursos naturales, uso de la tierra, clasificación de la vegetación, Reserva Nacional Pacaya-Samiria.

\begin{abstract}
The lowland peruvian Amazon remains sparsely populated and densely forested. Few roads exist and rivers provide much of the infrastructure. Over $12 \%$ of the area comprises flood plains inundated by the larger rivers, but due to their easy access and relatively fertile conditions, they provide a much larger share or the resources extracted in the region, and sustain most of the rural villages. The largest area of annually flooded land, constituting more than $60000 \mathrm{~km}^{2}$, surrounds the lower reaches of the Ucayali and Marañon river above their confluence to the Amazon proper, including almost $90 \%$ of the $20600 \mathrm{~km}^{2}$ large Pacaya-Samiria National Reserve. The entire area is constantly reshaped by erosion and deposition by the two main rivers, but also by smaller rivers which carry fewer sediments and less nutrients. The vegetation constitutes a complex mosaic of habitats defined by combinations of hydrological, physical, chemical and biological characteristics. Sixteen habitats including 12 forest formations are classified and described. Most flood plain inhabitants have lost their native identify, but they descend mostly from Amerindians rather than from recent immigrants, and preserve much knowledge on the flood plain environment and its habitats and uses. Their economic activities within the different forest formations are described. They combine agriculture, fishery, hunting and extraction of other forest products, and they market increasing amounts of these products. Growing populations and a increase in the need for monetary incomes as well as in external economic interventions, increasingly endanger economically important flood plain plants, animals and fishes. This is even the case within the Pacaya-Samiria National Reserve, making it urgent to develop and implement sound management systems in the marginal zones of the reserve, and elsewhere in Peruvian flood plains.
\end{abstract}

\title{
1. INTRODUCCIÓN
}

A una escala global, los pantanos cubren de 200 a 530 millones de hectáreas, aproximadamente el 3\% de la superficie terrestre. Cerca del $60 \%$ se encuentra cubierto con vegetación de bosque que recibe bastante agua fresca. Los trópicos poseen mucho más pantanos que las regiones boreales y templadas, y la mayor parte está cubierta de bosques (Lugo et al., 1990a). Mucho de los pantanos proveen servicios económicamente importantes para la pesquería, agricultura y forestería (Welcome, 
1985; Bayley, 1989; Bayley \& Petrere, 1989; Ros-Tonen, 1993; de Jong, 1995), y al mismo tiempo se constituyen en importantes hábitat para la vida vegetal y animal (Soini et al. ,1996; Junk \& Silva, 1997; Junk et al.,1997; Petermann, 1997), generalmente acentuando la necesidad de un manejo de múltiple propósito, adaptado a los ecosistemas y a la gente que interactúa con ellos.

Según Junk (1993) e Irion et al. (1997) los principales ríos de la cuenca amazónica poseen llanuras aluviales que cubren un área total de $300,000 \mathrm{~km}^{2}$. En la Amazonía peruana esta área incluye más de $60,000 \mathrm{~km}^{2}$ de tierras aluviales que están expuestas a inundaciones anuales de los ríos ricos en sedimentos que nacen en los Andes, y, en el Brasil, el mismo río Amazonas también rico en sedimentos inunda un área igual o mayor (Salo \& Räsänen, 1989; Junk, 1997).

La productividad de las llanuras aluviales que son inundadas por agua turbia, se incrementa debido a los nutrientes que se depositan durante la inundación anual (Furch, 1997), mientras que por otro lado, los periodos de inundaciones largas o irregulares disminuyen el crecimiento de las plantas (Gill, 1970; Junk, 1989; Irion et al., 1997). En la Amazonía, las condiciones de relativa fertilidad combinadas con patrones de inundaciones monomodales predecibles y el fácil acceso, hacen atractivas a las llanuras aluviales para actividades humanas y asentamientos poblacionales. En comparación, las áreas más extensas de tierra firme usualmente presentan suelos desgastados inadecuados para el cultivo permanente, y se encuentran ubicados más distantes de los ríos principales lo que dificulta el transporte. Consecuentemente, cuando llegaron los europeos, las llanuras aluviales estaban más densamente pobladas que tierra firme (Meggers, 1971; Denevan, 1976; Hemming, 1987; Roosevelt, 1989; Grenand \& Grenand, 1993; Moran, 1993). Inclusive hoy en día, la población rural de la Amazonía peruana continúa dependiendo de manera predominante de los recursos de la llanura aluvial (Hiraoka, 1985 a; Padoch, 1988; de Jong, 1995).

En el Perú, muchos de los habitantes de la llanura aluvial preservan un conocimiento íntimo del entorno de la llanura aluvial y de sus recursos (Hiraoka, 1985a; Padoch, 1988). Por ejemplo, manejan sistemas agrícolas adaptados a las llanuras aluviales (de Jong, 1995) y desarrollan cultivos específicamente adaptados a las llanuras aluviales (Sorensen et al.,1997). De esta manera, sus prácticas y experiencias tradicionales podrían brindar una visión valiosa para el manejo eficaz de la llanura aluvial, en el Perú o en cualquier parte. Esto se aplica especialmente a los dos grandes grupos étnicos ubicados en las llanuras aluviales del Perú, los Cocama-cocamilla y los ShipiboConibo. Sin embargo, la mayoría de los habitantes no-indígenas, localmente conocidos como "ribereños", también preservan muchas prácticas tradicionales que evidencia que son descendientes de las poblaciones indígenas (Padoch, 1988; Lonzoy, 
1992; San Román 1994). Tanto los nativos como los ribereños combinan sus actividades de subsistencia entre la agricultura, la pesca, la caza y la extracción de otros productos del bosque.

La disminución de valiosos recursos de la llanura aluvial, por efectos de la excesiva extracción, así como la pérdida de los hábitat, relacionado con la conversión del uso de la tierra, se constituyen como las mayores amenazas para sus poblaciones de plantas y animales. Los extractores de recursos penetran grandes distancias a través de los bosques inundados buscando entretenimiento, pesca o madera valiosa, que venden en crecientes cantidades en los mercados de la región. Es así que la mayoría de los bosques de la llanura aluvial del Perú han estado sometidos a extracciones para subsistencia, para fines comerciales, incluyendo extracción de madera a gran escala. Consecuentemente, ya han disminuido las poblaciones de un número de especies de animales y plantas que tienen valor comercial (Tabla 1 y 2 , respectivamente), lo que podría poner en riesgo su diversidad genética, dependiendo del tipo de uso (FAO, 1993 a; Namkoong et al., 1996). Es probable que se incremente más la extracción de algunos recursos de la llanura aluvial. Existe una población que va en aumento viviendo en las llanuras aluviales o cerca de ellas, que anhelan mejores niveles de vida que las generaciones anteriores. Por el momento, su mejor opción económica parece orientarse a la intensificación de la extracción de recursos de los bosques de la llanura aluvial, debido a que la producción agrícola de subsistencia es ineficiente, y a que, usualmente, es limitado el incremento de tierras agrícolas de primera.

Una producción agrícola más intensa y más extensa podría ser una alternativa para mejorar los intereses externos y el de los habitantes locales, que contraste con el cultivo actual diversificado de base familiar y de pequeña escala. A escala global, gran parte del bosque de llanura aluvial ya ha sido convertido en tierras para usos de agricultura (Lugo et al., 1990b), y en la Amazonía brasileña, grandes extensiones de bosques de llanura aluvial han sido deforestados a la altura de la parte media del río Amazonas hacia abajo y hacia arriba con respecto a la ciudad de Manaus (Goulding et al., 1996), principalmente para fines de pastoreo y ganadería (crianza de búfalos de agua) (Ohly, 1985). En el Departamento de Loreto, Perú, recientemente se hizo un llamado a la producción agrícola intensificada en la llanura aluvial debido a sus condiciones edáficas superiores en comparación con tierra firme (IIAP, 1997).

La excesiva extracción de valiosas especies forestales seleccionadas de la llanura aluvial, así como la conversión de extensos bosques a otros usos, podría ser un gran problema, ya que los ecosistemas de bosques de llanura aluvial son ecológicamente y socioeconómicamente importantes (Anderson, 1990; Peters, 1990; de Jong, 1995; 
Gram \& Kvist, en prensa). La disminución de ciertas especies puede poner en riesgo a su diversidad genética y a su aporte socioeconómico, y la conversión a gran escala de ecosistemas forestales podría acarrear enormes e impredecibles consecuencias a todo el entorno ambiental de la llanura aluvial. Sin embargo, es probable que la llanura aluvial del Perú aumente su tendencia a convertirse hacia la agricultura intensiva, ya que contiene los suelos más fértiles de la región amazónica (Furch, 1997; Junk, 1997).

Aunque los bosques por naturaleza están adaptados a algunas grandes perturbaciones (Foster et al., 1986; Foster. 1990a, 1990b; Worbes et al., 1992; Salo \& Kalliola, 1993; Worbes, 1997), las consecuencias de conversiones extensas son inciertas y deberían evitarse en lo posible. Por lo tanto, los sistemas de uso de la tierra deben desarrollarse de una manera que salvaguarden la biodiversidad de la llanura aluvial en donde fueron conducidos trabajos de campo.

El distrito municipal de Jenaro Herrera, localizado a lo largo de la parte baja del río Ucayali, a menudo sirve como un caso-ejemplo, y se hace referencia sobre experiencias realizadas en el canal de Puinahua, ubicado más arriba del río Ucayali, así como del río Marañón cerca de la confluencia con el afluente río Samiria. Estas áreas están todas localizadas dentro o cerca de la protegida Reserva Nacional Pacaya-Samiria, la cual abarca un área de casi $20600 \mathrm{~km}^{2}$, en donde la mayor parte consiste de bosques inundados localizados entre los cursos bajos de los ríos Ucayali y Marañón (Rodríguez et al., 1995).

\section{CLIMA Y GEOGRAFÍA}

Dourojeanni (1990), declaró que en el Perú aproximadamente $960000 \mathrm{~km}^{2}$ de ríos desaguan en el Amazonas, y que cerca de $770000 \mathrm{~km}^{2}$ pertenecen a la región amazónica. Esta última área incluye $90000 \mathrm{~km}^{2}$ de bosques montañosos tropicales ubicados en las vertientes andinas orientales, a $600 \mathrm{~m}$ de altura, dejando así aproximadamente $680000 \mathrm{~km}^{2}$ situados por debajo de los $600 \mathrm{~m}$ de altura, en donde la vegetación natural se compone de bosques húmedos tropicales bajos. Los departamentos de Loreto, Ucayali y Madre de Dios cubren un área de $556446 \mathrm{~km}^{2}$ que corresponden al $82 \%$ del total de la zona baja del Perú (Tabla 3). El resto del área se encuentra en los departamentos de Amazonas, San Martín, Huánuco, Pasco, Junín, Cuzco y Puno.

En base a estudios de imágenes de satélite, Salo \& Kalliola (1993) encontraron que solamente $0,8 \%$ de la Amazonía tropical baja del Perú fue deforestada durante 10-15 
años hasta 1993. También Gentry \& López (1980) y Dourojeanni (1990), observaron que la deforestación en los bosques amazónicos, sobre los $600 \mathrm{~m}$ de altura, se encuentra mucho más avanzada.

No existen cifras específicas que señalen la dimensión poblacional en la región baja de la Amazonía peruana. Sin embargo, en la Tabla 3, se indica que en 1993, cerca del 5\% de la población total del Perú (22 639 443) vive en los tres departamentos de la Amazonía baja: Loreto, Ucayali y Madre de Dios. Su densidad poblacional fluctúa de 0,8 a 3,2 habitantes por $\mathrm{km}^{2}$. Aproximadamente el $60 \%$ de la población de la Amazonía baja es urbana y vive predominantemente en las pocas ciudades grandes de la región: Iquitos, Pucallpa, Puerto Maldonado y Yurimaguas. El 40\% restante de habitantes rurales, mayormente se encuentran asentados a lo largo de los principales ríos que pasan por la llanura aluvial. Esto implica que aún quedan extensas áreas casi deshabitadas. Sin embargo, las poblaciones de los tres departamentos de la Amazonía baja, se han expandido a mayor velocidad que los promedios nacionales de $2,4 \%$ y $2,0 \%$ durante los periodos 1972-1981 y 1981-1993, respectivamente.

Salo y Rässänen (1989) estimaron que $62000 \mathrm{~km}^{2}$ de la Amazonía baja del Perú se encuentran expuestos a las inundaciones anuales, causadas por los principales ríos de sedimentos que nacen en las cumbres andinas y que vierten hacia la región (Salo et al., 1986). Además, extensas áreas se inundan por los ríos y quebradas locales que transportan sedimentos, o por las lluvias. Sumando estas áreas mencionadas que reciben pocos sedimentos, la llanura aluvial podría constituir hasta el $20 \%$ de la región de la Amazonía baja del Perú, correspondiente a la cifra estimada por Junk (19931997) para toda la zona baja tropical de Sudamérica. También existen áreas poco drenadas que se encuentran en tierras topográficamente altas y relativamente llanas, lejos de cualquier río (Encarnación, 1993), que aumentan más el porcentaje de áreas inundadas temporalmente o permanentemente en la Amazonía baja del Perú.

La llanura aluvial es extensa a lo largo del río Amazonas y de sus dos afluentes más grandes: el río Ucayali y el río Marañón. Más de $60000 \mathrm{~km}^{2}$ de bosques de llanura aluvial, lagos y pantanos se ubican principalmente al sur del río Marañón, rodeando los márgenes bajos de los Ríos Ucayali y Huallaga, así como de tributarios más pequeños como el río Maquía, río Tapiche, río Pacaya y el río Samiria (Rässänen et al., 1992, 1993). Esta extensa área inundada fue designada como el sub-valle PastazaMarañón por Rässänen et al. (1987), y como la depresión Ucamara por Villarejo (1988). Es el más grande entre cuatro sub-valles del oeste (Rässänen et al., 1987, 1990, 1993). Los otros tres son los sub-valles de inundación y deposición: Ucayali, Beni y Acre. El primero se localiza en el Perú rodeando al río Ucayali, aproximadamente desde río arriba de la ciudad de Contamana hasta el nacimiento de este tribu- 
tario, en la confluencia de los ríos Urubamba y Tambo; mientras que los otros dos sub-valles se localizan en Bolivia y Brasil, respectivamente.

Las temperaturas anuales promedios de la cuenca Amazónica fluctúan de $23^{\circ} \mathrm{C}$ a $27^{\circ} \mathrm{C}$, y las variaciones durante el año son pequeñas, aunque a veces el aire polar cubre el continente desde el sur y la temperatura cae a $10^{\circ} \mathrm{C}-15^{\circ} \mathrm{C}$. La precipitación varía de $1500 \mathrm{~mm}$ por año, en el sur y norte de la cuenca, a más de $6000 \mathrm{~mm}$ por año en algunas vertientes de los Andes, aunque el promedio es de $2000 \mathrm{~mm}$ a 3000 mm (Salati, 1985; Eden, 1990). En la Amazonía baja del Perú, la parte norte es la más húmeda, mientras que en el sur el clima es más seco y estacional (Kalliola \& Puhakka, 1993).

En Jenaro Herrera el clima típico es húmedo de zona baja tropical. La Figura 1 presenta un diagrama climático para el periodo 1970-1989. La temperatura mensual promedio es de $26,4^{\circ} \mathrm{C}$ con pequeñas fluctuaciones durante el año, y la temperatura diaria promedio es de $29,9^{\circ} \mathrm{C}$. La precipitación anual promedio es de 2687 $\mathrm{mm}$ con variaciones anuales en el rango de $1700-4000 \mathrm{~mm}$ aproximadamente. No se observa estaciones secas o lluviosas pronunciadas, aunque los meses de junio a octubre normalmente son menos lluviosos que los meses de noviembre a mayo. La variación entre las precipitaciones mensuales oscila de 54 a $758 \mathrm{~mm}$ (Claussi et al., 1992). Durante la estación más soleada de junio a setiembre, hay hasta 200 horas de sol por mes, mientras que de febrero a abril esta cantidad se reduce a 100 horas (Gautier \& Spichiger, 1986).

\subsection{Tipos de inundaciones y clases de agua}

Para los ecosistemas pantanosos el tipo de inundación es un factor medioambiental importante. Las inundaciones pueden ocurrir más o menos de forma regular, tener diferentes amplitudes, y durar cortos o largos períodos. Los períodos de inundación de la llanura aluvial de los ríos grandes, a menudo siguen modelos monomodales predecibles, mientras que en la llanura aluvial a lo largo de quebradas y ríos pequeños así como en pequeñas depresiones, las inundaciones tienden a ser polimodales impredecibles. En las depresiones mayores y en las áreas de poco drenaje, pueden al contrario ocurrir inundaciones predecibles de baja magnitud y muy vinculadas a las características de lluvia (Junk, 1997). En todos los casos, la llanura aluvial debería ser vista como una unidad del río ecológicamente indivisible y estrechamente vinculada al mismo así como a su área de captación, según el concepto básico de inundación de Junk et al. (1989). 
El mismo río Amazonas así como sus tributarios mayores, se caracterizan por tener un ritmo de inundación de tipo monomodal anual. Junk $(1984,1989) \&$ Irion et al. (1997) presentaron información sobre los tipos de inundaciones a diferentes estaciones de medición en Brasil. En Manaus, encontraron que la magnitud promedio de inundación era de 9,95 m durante un periodo de 90 años, con la curva más elevada en el mes de Junio y el nivel de agua más bajo cerca de octubre. Recalcan las implicaciones ecológicas de la inundación relativamente predecible, en cuanto al tiempo de los niveles mínimo y máximo del agua, así como al hecho de que durante varios años consecutivos puede ocurrir con niveles máximos o mínimos de agua.

En la Figura 2a, se muestran los promedios mensuales de niveles de agua tomados mediante medidas diarias, de setiembre de 1987 a febrero de 1997, en el río Amazonas cerca de Iquitos, y en la Figura 2b, se presentan los correspondientes registros realizados en el río Ucayali cerca de Jenaro Herrera. También se incluyen los valores máximos y mínimos registrados cada mes durante el mismo periodo. En ambos lugares, el período promedio de máximo nivel ocurrió de marzo a mayo y los niveles más bajos se registraron de agosto a octubre, pero durante algunos años, los niveles de agua máximo y mínimo se registraron antes o después de los periodos anteriormente mencionados. El nivel máximo alcanzado en Jenaro Herrera es en promedio mayor que el de Iquitos, y también ocurre antes, lo cual podría deberse a que Jenaro Herrera está localizado aguas arriba respecto a Iquitos. En ambos casos, el tope máximo del nivel de agua sugiere una correlación con la cantidad de precipitación del lugar (Figura 1), aunque las fluctuaciones del río probablemente dependen más de los tipos de lluvias que ocurren en las vertientes andinas más distantes. Durante el nivel máximo de agua, calculamos que más del $90 \%$ de la llanura aluvial se encuentra inundada, mientras que en la estación de merma, usualmente menos del $10 \%$ permanece cubierto de lagos, pantanos y ríos permanentes. Sin embargo, durante algunos años (p. ej. en 1993 y 1994) casi se inundó toda la llanura aluvial, a pesar que el nivel de agua en esos años raramente llegaba a un metro y medio por encima del nivel máximo de los años promedio. De todas maneras se inundan los sitios más elevados de la llanura aluvial, debido a que los mismos se formaron por depósitos ocurridos durante las anteriores inundaciones más altas, y su altura en consecuencia corresponde al nivel máximo de agua. Cuando ocurren tales inundaciones, las cosechas y árboles sensibles sufren daños o pueden morir, y también se pueden ahogar los animales que habitan en la llanura aluvial.

Gentry \& López anunciaron un incremento en los máximos niveles anuales de agua en Iquitos, durante el periodo 1962-1978, debido a la deforestación ocurrida en los Andes, pero según Nardin \& Meade (1982) sus datos fueron interpretados muy 
pobremente. La Figura 3 muestra los niveles anuales de agua, máximo y mínimo, registrados en Iquitos durante el periodo 1980-1997. En este periodo no encontramos ningún indicio de aumento en el nivel máximo de inundación a pesar que la deforestación de las vertientes andinas viene ocurriendo desde 1978 a la fecha.

Se distinguen tres tipos principales de ríos amazónicos, según sus aguas, que se nombran en bases a sus colores característicos (Sioli, 1968, 1984; Schmidt, 1972; Junk, 1983; Eden, 1990). Los ríos de "agua blanca" contienen grandes cantidades de sedimentos en suspensión, así como una considerable concentración de nutrientes, y su reacción es casi neutral. Los ríos de "aguas negras" ganan su coloración debido a sustancias fenólicas que se degradan lentamente, son pobres en nutrientes y su reacción es ácida. Los ríos de "aguas claras" no contienen concentraciones significativas de sedimentos en suspensión ni materias orgánicas, por lo tanto, son más transparentes que los ríos de agua blanca y negra, mientras que su reacción puede variar considerablemente. Además, algunos investigadores consideran a los ríos de "aguas mezcladas" como una categoría aparte (Encarnación, 1985, 1993). Este término define al agua negra o clara mezclada con agua blanca.

Los ríos y quebradas de agua clara provienen de áreas de roca firme, geológicamente muy antiguas, y que han sido altamente lixiviadas. Estos tipos de ríos son un poco raros en el Perú, mientras que algunos de los grandes tributarios brasileños son del tipo de agua clara, por ejemplo, el río Xingú y el río Tapajós que nacen en la parte central del Brasil (Edén, 1990). Los ríos y quebradas de agua negra son comunes en el Perú aunque no se cuentan entre los grandes ríos. Nacen de áreas arenosas sujetas a podzolización, y de áreas pantanosas muy poco drenadas (Encarnación, 1993). Los dos tributarios, el río Pacaya y el río Samiria, que dan el nombre a la Reserva Nacional Pacaya-Samiria, son del tipo de agua negra, igual que el río Nanay que desemboca en el Amazonas cerca de la ciudad de Iquitos (Encarnación, 1993). En contraste, los grandes ríos de agua blanca dominan el paisaje en la Amazonía peruana. A esta categoría pertenece el mismo Amazonas, el Napo, el Marañón, el Huallaga, el Ucayali y el Madre de Dios. Todos estos ríos nacen en las montañas andinas y arrastran sedimentos erosionados de sus vertientes y también aluvión disuelto, en segundo lugar, de las cuencas de los ríos de la zona baja (Edén, 1990). Sin embargo, los tributarios cuyas cabeceras completas se encuentran en las tierras bajas de la Amazonía peruana son también del tipo de agua blanca (Rässänen, 1993), ya que mayormente drenan paisajes geológicamente jóvenes y de fácil erosión cuya consistencia se compone de sedimentos depositados por el crecimiento del río (Rässänen et al., 1987, 1993). Esto contrasta con los paisajes antiguos y altamente lixiviados, drenados por los ríos de agua clara de la zona central del Brasil y de Guyana. 
La categoría de agua mezclada es importante en la Amazonía peruana. Los tributarios más grandes pueden recibir en sus cabeceras agua clara, negra y blanca de diferentes quebradas, y de esta forma quedar técnicamente caracterizados como mezclados, pero, en la práctica, se refiere a esos ríos de acuerdo a la categoría del color con el que sus aguas corresponden mejor. El término agua mezclada más bien se aplica a los lugares en donde el tipo de agua puede cambiar durante el año ya sea por estación o de forma irregular. Por ejemplo, muchas quebradas locales presentan agua negra durante la estación de vaciante y agua blanca durante los meses más lluviosos del año, lo cual prueba que el aumento de la corriente en esta época los vuelve más erosivos (Encarnación, 1993; Rässänen, 1993).

Cerca de Jenaro Herrera se observó este cambio, en el bosque Lobillo, el cual se inunda anualmente cerca de la confluencia de la quebrada de agua negra Aucayacu y del canal de llanura aluvial de Lobillo que conecta con el río Ucayali. Las tormentas de lluvia del lugar aumentan la descarga del canal de Aucayacu y entonces el bosque Lobillo se inunda con agua blanca, que proviene de las subidas de nivel del río Ucayali. El agua del río fluye a través del canal dentro del bosque Lobillo depositando considerables cantidades de sedimentos.

Dentro de la Reserva Nacional Pacaya-Samiria, las aguas se mezclan y se alternan en gran escala. La mayoría de los lagos y pantanos de la Reserva reciben agua negra de los ríos y quebradas del lugar, excepto en periodos que vienen después de la subida de los niveles de agua en los ríos aledaños Ucayali y Marañón. Durante estos períodos, el agua blanca puede ingresar a la Reserva por días o semanas, especialmente desde el río Ucayali ingresando a través de estrechos canales y por el río Pacaya que usualmente es de agua negra. Inclusive la corriente del río Pacaya puede cambiar temporalmente de dirección y llevar río arriba agua blanca o mezclada en vez de agua negra hacia río abajo (Räsänen et al., 1993). Los extensos lagos, pantanos y canales dentro de la Reserva Nacional Pacaya-Samiria están interconectados con el río Ucayali y con el río Marañón. Los pescadores pasan en canoas a través de la Reserva entre estos dos ríos principales, y el agua puede fluir hacia los numerosos cuerpos de agua de la Reserva, dependiendo de las fluctuaciones de los dos ríos principales así como de las quebradas y otros ríos de la zona.

\subsection{Procesos fluviales y formas de paisajes}

Los procesos fluviales continuamente afectan el paisaje de la llanura aluvial, a diferentes escalas en el tiempo y el espacio, influenciando de manera profunda las condiciones medioambientales y creando diferentes formas de paisaje. Es difícil deter- 
minar la extensión de los ecosistemas de la llanura aluvial que toman parte en este proceso, aunque Junk et al., (1989) los definen como áreas sujetas a inundación periódica, que generan adaptaciones y el desarrollo de estructuras comunitarias típicas. El cinturón de meandro se compone de áreas sujetas a la migración activa del río a lo largo del canal, mientras que las cuencas inundables se ubican más allá del canal del río, y tienden a tener un terreno topográficamente más bajo, de poco drenaje y a menudo pantanoso en climas húmedos. El agua del río inunda el cinturón de meandro mientras que la cuenca aluvial se inunda con el agua de pequeños ríos del lugar y de la lluvia (Reineck \& Singh, 1980; Kalliola \& Puhakka, 1993; Junk, 1997). La mayor parte del paisaje que se encuentra en el sur y hacia el este del subvalle Pastaza-Marañón (la depresión Ucamara) está formada por el río Ucayali, y varios tributarios más pequeños siguen sus antiguos cinturones de meandro, por ejemplo, los ríos Tapiche, Pacaya y Samiria (Dumont \& García, 1992). El actual cinturón de meandro de la parte baja del río Ucayali tiene de 10-20 km de ancho (Kalliola et al., 1992). En la región de Jenaro Herrera, hacia el oeste, está bordeado por tierras altas que no se inundan y, hacia el este, por extensas cuencas aluviales (Dumont et al., 1990), pero aguas arriba de Requena su cinturón de meandro está rodeado de cuencas aluviales por ambos lados.

Los ríos constituyen la fuerza motriz en los procesos fluviales. En la Amazonía baja del Perú los canales de los ríos toman formas de meandro, anastomosis y trenzas. El río Ucayali es un ejemplo de río de muchos meandros, que se distingue por poseer un solo canal muy sinuoso. Además de tener pocos meandros, el río Marañón es en parte anastomosizante e inclusive trenzado, y se caracteriza por poseer varios canales, y por ser de alta y baja sinuosidad respectivamente. La forma serpenteante que probablemente sea la forma más común de los grandes ríos de la Amazonía peruana, se ve favorecida por pendientes bajas, material cohesivo de las orillas, nivel elevado de carga en suspensión dentro del cauce y un flujo de agua relativamente estable (Reineck \& Singh, 1980, Puhakka et al., 1992; Rässänen et al., 1992; Kalliola \& Puhakka, 1993).

La erosión y la sedimentación son procesos básicos de la dinámica del río. En los ríos serpenteantes, la erosión ocurre predominantemente al exterior de los meandros, ocasionando la migración lateral del río. Cuando el canal de un río migra hacia un nuevo curso, ocurren cambios repentinos y dramáticos durante el recorrido del río. Este proceso conocido como avulsión del río (separación violenta) puede ser causado por el aumento gradual del terreno en el cinturón de meandro debido a la sedimentación (Reineck \& Singh, 1980; Rässänen et al., 1992). El efecto de la erosión está ilustrado por las condiciones del río Ucayali. Al sur de Requena, el canal del río migra hasta $160 \mathrm{~m}$ cada año (Kalliola et al.,1992), mientras que en Jenaro 
Herrera la erosión es más lenta (de Jong, 1995). Las avulsiones del río ocurren repentinamente, por ejemplo en la mitad del río, donde hace unos 200 años más de $50 \mathrm{~km}$ de canal se reubicaron hasta $80 \mathrm{~km}$ (Parssinen et al., 1996). Igualmente, el canal del Puinahua probablemente representa a una reciente avulsión del río y aún posee un cinturón de meandro estrecho y topográficamente bajo rodeado de extensos pantanos (Kalliola et al., 1991a). En el último caso, continua fluyendo bastante agua por el antiguo canal del río Ucayali.

La sedimentación ocurre en diferentes partes de la llanura aluvial, y para fines prácticos podemos dividirla en tres grandes grupos de depósitos: (1) depósitos del canal, (2) depósitos de las orillas, (3) depósitos de la cuenca aluvial (Reineck \& Singh, 1980; Kalliola \& Puhakka, 1993).

Los depósitos del canal son de interés especial en los ríos serpenteantes, en donde crean depósitos de barrera que forman escollos continuos en las curvas del cauce. Los depósitos de barrera se desarrollan como formaciones largas y angostas en los lados internos de los meandros y paralelos a los mismos, y sus proporciones son de acuerdo al tamaño del río y se caracterizan por presentar una sucesión de materia espesa en la parte de abajo y de materia fina en la parte de arriba (Reineck \& Simgh, 1980). Luego, las barreras de las inundaciones aparecen temporalmente como playas, las cuales son colonizadas en pocos meses, inicialmente por malezas anuales. En cada inundación los sedimentos aumentan la altura de las barreras, eventualmente convirtiéndolas en escollos que se estabilizan por una vegetación perenne de hierba, arbustos y pequeños árboles. Los escollos están separados por depresiones angostas que se rellenan con sedimentos finos (Reineck \& Singh, 1980; Puhakka et al., 1993).

En el río Ucayali, los escollos tienen aproximadamente 6 metros de altura y se extienden de 2 - $4 \mathrm{~km}$. Están separados por hendiduras que son de 2-5 $\mathrm{m}$ más bajos que los escollos (Rässänen et al., 1992). En los sistemas de ríos serpenteantes, los depósitos que rellenan el canal, también rellenan los canales de ríos abandonados, mediante los procesos de separación o avulsiones y rápidamente quedan sellados cerca del canal del río activo, mientras que en los lagos cortados, la sedimentación ocurre mediante un proceso lento (Reineck \& Singh), 1980).

A lo largo de las orillas del río se extienden depósitos de sedimentos en forma de diques o grietas. Los diques son topográficamente los puntos más elevados de la llanura aluvial, principalmente ubicados en la parte externa del meandro o a lo largo de los cursos rectos del río, formando superficies grandes y planas. Las extensiones de grietas están formadas por los sedimentos que se depositan a lo largo de 
los atajos del canal a través de diques o bancos naturales, especialmente en la parte extensa de los meandros. En ambos casos, los sedimentos se depositan a niveles altos de agua, cuando disminuye la velocidad del agua al inundarse las riberas del río.

Los diques pueden bloquear el drenaje de extensas áreas que se encuentran detrás de ellos, ocasionando la destrucción del bosque y la formación de pantanos o de bosques pantanosos (Reineck \& Singh, 1980; Kalliola \& Puhakka, 1993). En la Amazonía baja del Perú, las superficies más altas de la llanura aluvial probablemente se originan de los depósitos ribereños. Los depósitos de la cuenca aluvial son resultado de la acumulación de sedimentos que sobrepasan las riberas del río para depositarse más allá del curso del río, particularmente en las áreas de la cuenca aluvial.

Estos sedimentos son pocos y muy finos, ya que las partes espesas tienden a depositarse más cerca del río. Las aguas de los ríos y quebradas del lugar también penetran las cuencas aluviales, y también arrastran pocos sedimentos finos. Sin embargo, se sabe que en áreas inundadas de agua negra se da una sedimentación anual de varias toneladas de sedimentos finos por hectárea (Walker, 1995), y con el tiempo los sedimentos depositados poco a poco recubren el relieve de las cuencas aluviales. Por lo tanto, las cuencas aluviales se modifican más lentamente que los cinturones de meandros.

Los procesos fluviales arriba descritos forman patrones complejos de paisaje en la llanura aluvial. Los diversos procesos actúan de forma diferente en el tiempo y el espacio, formando un paisaje variado de diferentes hábitat interconectados. Salo \& Rässänen (1989) clasificaron los procesos geomorfológicos que influyen en el tiempo y el espacio, los patrones del paisaje de la llanura aluvial y sus respuestas bióticas. Los procesos fluviales constituyen un factor principal en este proceso, en los que la sedimentación y la formación de barreras fluviales, meandros, diques, grietas y canales aluviales actúan dentro de una escala de 1-100 años, y se supone que tengan una extensión de hasta 100 hectáreas. En periodos más largos de 100 a 10000 años, se supone que los canales, las avulsiones, los cambios de rumbo del drenaje, los cambios en los tipos de canales y la formación de terrazas ejercen influencia sobre áreas más extensas. La formación de las cuencas suceden en periodos de millones de años y llega a cubrir vastas áreas. Las respuestas biológicas a estos procesos ocasionan la formación de diferentes comunidades vegetales, diferenciadas según la etapa de sucesión y condiciones medioambientales. Por ejemplo, los actuales bosques de meandros podrían haberse establecido en forma de vegetación colonizadora sobre diques o meandros de reciente formación, mientras que los bosques maduros de meandros son raros o no existen, lo que corresponde a la situación descrita por varios autores 
(p. ej. Foster et al., 1986; Foster, 1990 a: Gentry \& Terborgh, 1990; Worbes et al., 1992; Kalliola \& Puhakka, 1993, 1993; Worbes, 1997). En el río Ucayali, el índice de cambio de sitio que es el porcentaje anual promedio de erosión del cinturón de meandro, se calculó en aproximadamente 0,3\% (Kalliola et al., 1992). En el río Marañón, el correspondiente valor probablemente sea el mismo. Por ejemplo, en 1996, el río erosionó más de $500 \mathrm{~m}$ de tierra en su confluencia con el río Samiria, obligando a la gente de San Miguel a reubicar sus viviendas una y otra vez. Los índices de cambio de sitio de los valores mencionados, sugieren que grandes porciones de la vegetación del cinturón de meandros de la región del Pacaya-Samiria desaparecen por erosión en el transcurso de unos cientos de años. Por lo tanto, los bosques maduros o antiguos del cinturón de meandros son raros o ya no existen.

\subsection{La vegetación}

Para poder realizar una extracción eficaz, así como para efectuar un manejo adecuado, se requiere contar con una visión panorámica en cuanto a la distribución y disponibilidad de los recursos. Por lo tanto, se hace necesario separar diferentes sitios según la composición y estructura de la vegetación, aunque eso implique una serie de dificultades (Greig-Smith, 1983). Esto se puede ilustrar por las diferenciaciones entre los tipos de vegetación que suele hacer la gente que subsiste de los recursos naturales. De ninguna manera, la llanura aluvial amazónica es una excepción, ya que los diversos hábitat de diferentes tamaños y muchas otras zonas transitorias aparecen interconectados (Worbes et al., 1992; Puhakka \& Kalliola, 1993; Toumisto, 1993).

Según Klinge et al., (1990) para clasificar la vegetación de la llanura aluvial, es útil considerar: (1) los criterios hidrológicos, (2) los criterios físico-químicos, y (3) los criterios biológicos. Se pueden identificar varios indicadores para cada uno de estos criterios.

Entre los criterios hidrológicos, particularmente los aspectos del patrón de inundación, con énfasis en la duración de la inundación, se presentan como un factor decisivo para determinar la composición de la vegetación y la estructura de los bosques pantanosos en general (Bacon, 1990; Lugo et al.; 1990b; Junk, 1993), así como para los bosques de la llanura aluvial amazónica (Worbes, 1983, 1986, 1997; Junk, 1989, 1997; Klinge et al.,1990; Worbes et al., 1992; Kalliola \& Puhakka, 1993; Junk \& Piedade, 1997). Los estudios muestran que la tolerancia de los árboles a la inundación defiere según la especie y según el tamaño de los árboles, y que existen diferentes adaptaciones fisiológicas y anatómicas de las plantas a la inundación (Gill, 1970; Crawford, 1982; Hook, 1984; Kozlowski, 1984; Junk, 1989; Schluter et 
al.,1993; Armstrong et al., 1994; Worbes, 1997). Además, los patrones reproductivos se pueden adaptar al entorno inundado (Gottsberger, 1978., Goulding, 1980, 1987; Ziburski, 1991; Ayres, 1995; Kubitski \& Ziburski, 1994; Worbes, 1997). Sin embargo, eventos raros tales como varios años consecutivos de niveles de inundación altos y mínimos pueden contribuir con mayor fuerza al desarrollo de vegetación que el patrón promedio de inundación (Junk, 1989; Irion et al., 1997; Junk \& Piedade, 1997). Indicadores como la amplitud, la frecuencia y la duración de la inundación pueden, en consecuencia, ayudar a la clasificación de la vegetación de la llanura aluvial.

El tipo de agua que inunda la llanura aluvial, ejerce una fuerte influencia en las características químicas de sus suelos (Furch, 1997), y la erosión y la deposición del río dan forma al paisaje (Salo \& Rässänen, 1989). De esta manera, los indicadores químicos y físicos relativos a las características del suelo, del agua y a la forma del paisaje, pueden servir para clasificar la vegetación de la llanura aluvial (Prance, 1979, 1980; Encarnación, 1985, 1993; Pires \& Prance, 1985; Kubitzki, 1989).

Entre los criterios biológicos, la etapa de sucesión de la vegetación puede ser útil para clasificar la vegetación de la llanura aluvial. Varios autores opinan que la composición florística y la estructura de los bosques de la llanura aluvial están correlacionadas con este factor (Salo et al., 1986; Foster et al., 1986; Foster, 1990a; 1990b; Worbes et al., 1992; Worbes, 1997). Además, familias de plantas y especies características, pueden caracterizar diversas formaciones de bosques de llanura aluvial (Prance, 1979, 1980; Pires \& Prance, 1985; Campbell et al., 1986; Balslev et al.,1987; Worbes et al., 1992; Freitas, 1996; Worbes, 1997, Nebel et al., en esta edición).

Basado en las experiencias de los bosques brasileños, Prance $(1979,1980)$ efectuó la clasificación más ampliamente divulgada de la vegetación de los bosques amazónicos aluviales y pantanosos. Basó su terminología en la regularidad de la inundación y en los tipos de agua de inundación, sin considerar la etapa de sucesión de la vegetación. La principal distinción la hizo entre la várzea que se inunda de agua blanca e igapó que se inunda de agua negra o clara. La várzea del Brasil, según Prance (1979), se parece al tipo de bosque que en el Perú se conoce como tahuampa. Los tipos de bosques de igapó que se encuentran en el Brasil, muy difícilmente podrían existir en el Perú, debido a que los ríos y suelos del Perú raramente son tan pobres en nutrientes como lo son en la mayor parte de la Amazonía brasileña.

Varios investigadores hicieron una descripción de la vegetación de las llanuras aluviales amazónicas fuera del Perú (Black et al., 1950; Takeuchi, 1962; Braga, 1979; Keel \& Prance, 1979; Worbes et al., 1992; Ayres, 1995; Jung, 1993; Macedo 
\& Anderson, 1995). La mayoría de estos estudios fueron conducidos en la Amazonía central cerca de Manaus. Junk \& Piedade $(1993,1997)$ describieron la vegetación herbácea de la región.

En cuanto a la vegetación de los bosques de llanura aluvial de la Amazonía peruana, Toumisto (1993) comparó las clasificaciones existentes. Los trabajos más citados son los de Malleux (1971, 1975 citado por Toumisto, 1993) y Encarnación (1993). Malleux centró su atención en el potencial maderero de varios tipos de bosques y basó su clasificación en fotografías aéreas. Encarnación (1993), por su lado, basó su clasificación en un extenso trabajo de campo, y los tipos de vegetación y terminología reconocida y aplicada por la gente del lugar. Además, varios otros investigadores contribuyeron con valiosa información que puede ayudar a definir y a describir los tipos de vegetación de la llanura aluvial peruana, particularmente Kalliola et al. (1991a) respecto a vegetación de pantanos, Seidenschwarz (1986) así como Kalliola et al. (1991b) en lo que se refiere a vegetación colonizadora, y Kahn \& Mejía (1990) respecto a bosques dominados por palmeras. La vegetación madura, así como los aspectos de la sucesión de la vegetación de la llanura aluvial, fueron descritos por diversos autores (Salo et al., 1986; Gentry, 1988; Foster, 1990a, 1990b; Gentry \& Ortíz, 1993; Puhakka \& Kalliola, 1993; Puhakka et al., 1993; Freitas, 1996; Nebel et al., en esta edición). Basado en estudios de mapas, López \& Freitas (1990) examinaron y categorizaron bosques en la región de Jenaro Herrera en regular medida, poniendo en práctica la clasificación de Encarnación (1985). Lamotte (1990) describió las relaciones entre las dinámicas fluviales y la vegetación del bosque en una isla de la región de Jenaro Herrera.

En la Tabla 4, clasificamos algunos hábitat importantes de la llanura aluvial del Perú, ejemplificados por las áreas circundantes a la parte baja de los ríos Marañón y Ucayali, que corresponden al sub-valle del Pastaza-Marañón (la depresión Ucamara). La clasificación se basa en el trabajo de Encarnación $(1985,1993)$, buscando darle consistencia con la terminología empleada por la gente del lugar. Los indicadores usados para este fin son: el período de inundación, las características del agua inundante, el drenaje del suelo, la ubicación geográfica de acuerdo al principal curso del río, la estructura de la vegetación, la etapa de sucesión de la vegetación y la presencia de plantas características. Estos indicadores representan a los tres grupos de criterios mencionados arriba y ayudan a identificar 16 formaciones de vegetación. Hay que tomar nota que la distinción entre las etapas de sucesión inicial y posterior, se restringe al cinturón de meandro. Aquí las perturbaciones de la vegetación ocasionadas por los procesos dinámicos del río, ocurren con más frecuencia que en las áreas de la cuenca aluvial, haciéndose necesario clasificar las etapas de la vegetación joven. Además, la población local extrae más recursos de los hábitat del 
cinturón de meandro (ver Tabla 6), y, por lo tanto, aplica una terminología más detallada a estas formaciones, en comparación con las cuencas aluviales más distantes y generalmente menos fértiles. El límite entre las formaciones vegetales es gradual, y mucha vegetación puede tener caracteres intermedios.

Se entiende por restinga a la parte alta de la llanura aluvial, la cual sólo se inunda en algunos años o por un periodo más corto. Encontramos que los bosques de restinga en promedio se inundan durante tres meses cada año, y se considera conveniente distinguir entre restinga alta y baja, las cuales se ubican en lugares que se inundan en promedio anual por 1 mes y de 1-3 meses respectivamente. Los bosques de restinga son altos, cerrados y diversificados, y se distinguen seis categorías $\left(\mathrm{N}^{\circ} 1,2,3,4,7 \mathrm{y}\right.$ 8 en la Tabla 4). Los bosques de restinga alta $\left(\mathrm{N}^{\circ} 1, \mathrm{y} 7\right)$ tienden a situarse en superficies planas y relativamente grandes, ya que típicamente se originaron de depósitos ribereños. Por el contrario, la restinga baja $\left(\mathrm{N}^{\circ} 3\right.$, y 8$)$ a menudo aparece en tierra creada por los depósitos del canal y en típica forma de meandro, compuesto de largas y estrechas aristas cortadas por bajiales. Las restingas productos de deposiciones recientes en el cinturón de meandros, se designan como jóvenes $\left(\mathrm{N}^{\circ} 2\right), \mathrm{y}$ ciertas valiosas especies pioneras como la Calycophyllum spruceanum (Benthan) Hooker f. ex Schumann y Ficus insípida Willd. tienden a ser prevalecentes aquí, ya que se regeneran en condiciones abiertas. En otros bosques maduros de restinga $\left(\mathrm{N}^{\circ}\right.$ 1, 3, 7 y 8), que se caracterizan por la dinámica de espacio-fase (Foster et al., 1986; Worbes et al., 1992; Worbes, 1997), estas especies a menudo están ausentes o solamente representadas por árboles grandes. Los bosques de restinga joven pueden ser relativamente similares, independientemente de la duración del período de inundación. Tradicionalmente se desarrollan cerca del río, en lugares donde considerables cantidades de sedimentos se depositan durante la inundación, y de esta forma su terreno crece rápidamente, siendo necesario que los árboles retengan estos sedimentos para poder sobrevivir. Debido a la migración del río, la mayoría de los bosques del cinturón de meandro son jóvenes, mientras que los bosques de restingas de la cuenca fluvial $\left(\mathrm{N}^{\circ} 7\right.$ y 8$)$ que se ubican lejos de los cursos actuales del río principal, podrían tener miles de años. En la región del Pacaya-Samiria la mayoría de las restingas de la cuenca aluvial fueron depositados por los anteriores cursos de los ríos Ucayali y Marañón. Estas áreas se pueden catalogar como cinturón de meandro antiguo.

La tahuampa ( $\mathrm{N}^{\circ}$ 5, 6 y 9 en la Tabla 4), se sitúa en terreno más bajo que la restinga, y en consecuencia se inunda por un período mayor, cuya duración anual promedio va de 3 a 6 meses. Sin embargo, la tahuampa, usualmente se drena muy bien durante los meses restantes del año. Los bosques de tahuampa madura $\left(\mathrm{N}^{\circ} 5\right)$ se distinguen de la tahuampa joven recientemente establecida $\left(\mathrm{N}^{\circ} 6\right)$, en base a la presencia o ausencia 
de especies de temprana sucesión. La tahuampa de la cuenca aluvial $\left(\mathrm{N}^{\circ} 9\right)$ tiende a situarse lejos de los cursos actuales de los ríos principales, es más pobre en nutrientes y mucho más antigua que la tahuampa del cinturón de meandro $\left(\mathrm{N}^{\circ} 5\right.$, y 6$)$.

Grandes extensiones de la llanura aluvial cubierta de vegetación maderable drenan muy poco ( $\mathrm{N}^{\circ} 10,11,12$ y 13 en la Tabla 4), por esta razón la tala de árboles se hace antes del final de la inundación anual y después de las lluvias torrenciales. Muy pocos árboles crecen más en áreas inundadas, sometidas a explotación forestal irregular o permanente (Junk, 1989), que en áreas que se caracterizan por tener inundaciones anuales regulares, y a menudo prolongadas, es decir, restinga y tahuampa. Es por eso, que los bosques de áreas poco drenadas tienden a ser de baja estatura, atrofiados, iluminados, pobre en especies y en consecuencia pueden tener mucha hierba y lianas en la parte baja. Los bosques pantanosos pueden estar compuestos de una mezcla de especies de dosel $\left(\mathrm{N}^{\circ} 12\right.$ y 13). Los bosques de bajial ( $\left.\mathrm{N}^{\circ} 10\right)$, típicamente se encuentran cerca de los ríos principales en el cinturón de meandro inundado de agua blanca. El sotobosque usualmente es muy denso y casi impenetrable debido a densas poblaciones de hierbas grandes (Calathea, Heliconia y Ischnosiphon) y muchas lianas y árboles caídos. Los bajiales pueden ser largas y estrechas depresiones (swales) de complejas fajas o constituir extensas áreas separadas de los ríos por diques naturales que bloquean su drenaje. Los bosques de bajial de la cuenca aluvial $\left(\mathrm{N}^{\circ} 11\right)$ poseen un dosel florísticamente mezclado y surgen en áreas de la cuenca aluvial que se inundan de agua negra o mezclada, que proviene de los ríos y también de la lluvia. Por lo tanto, las condiciones tienden a ser más pobres en nutrientes y ácido que en los bosques de bajial $\left(\mathrm{N}^{\circ} 9\right)$. El aguajal $\left(\mathrm{N}^{\circ} 13\right)$ recibe su nombre de la palmera del aguaje Mauritia fleuxosa L. f, que predomina en extensas áreas inundadas de explotación forestal, especialmente localizadas en las cuencas aluviales. Se inunda con agua de los ríos de agua negra o mezclada y por agua de lluvia. En muchos, aunque no en todos los aguajales, las palmeras Euterpe precatoria Mart. y Oenocarpus mapora H. Karst. también son sus principales constituyentes. El Pungal ( $\left.\mathrm{N}^{\circ} 12\right)$ gana su nombre del predominante árbol "punga", Pseudobombax munguba (C. Martius \& Zuccarini) Dugand, que pertenece a la familia Bombacaceae. A menudo predomina en los bosques pantanosos, pero tiende a crecer en lugares más ricos en nutrientes que la palmera del aguaje, especialmente en la parte intermedia entre otros tipos de bosques y pantanos que rodean ríos y lagos de la llanura aluvial, que corresponden al pantanal, al lago y al río ( $\mathrm{N}^{\circ} 14$, y 15 en la Tabla 4). La mayoría de los lagos surgen en antiguos lechos de río de meandros separados, y la vegetación del pantano de raíces firmes en vez de vegetación flotante, se va desarrollando mientras los sedimentos se van depositando. Por consiguiente, el árbol punga invade formando un pungal, el cual más adelante puede ser reemplazado ya sea por 
una tahuampa, un bajial o un aguajal, dependiendo de las condiciones del lugar. El barrial y la playa $\left(\mathrm{N}^{\circ} 16\right.$ y 17 de la Tabla 4) están situados a lo largo de los ríos principales y surgen por la deposición de barro y arena respectivamente. Las sucesiones que ocurren en estos lugares forman los bosques de restinga, tahuampa o bajial.

\subsection{Población: Historia, cultura y demografía}

Los primeros exploradores españoles y portugueses informaron que la llanura aluvial a lo largo del río Amazonas estaba densamente poblado, por grandes y numerosas aldeas de amerindios, organizados en dominios caciquistas que se extendían a lo largo de cientos de kilómetros de río (Meggers, 1971; Denevan, 1976; Moran, 1987; Roosevelt, 1989). Sin embargo, las epidemias traídas de Europa así como la captura de esclavos iniciada por los europeos, en poco tiempo redujo las poblaciones amerindias, y los que sobrevivieron fueron trasladados y asentados en poblados misioneros donde usualmente los diferentes grupos étnicos se mezclaban entre sí (Hemming, 1987; Parker, 1989).

En Brasil, hace más de doscientos años que las últimas culturas amerindias de la llanura aluvial perdieron su lengua nativa, y desde entonces sus descendientes hablan portugués (Ross, 1978; Hemming, 1987; Grenand \& Grenand, 1993). A estos descendientes se les conoce actualmente como "caboclos", pero aún conservan mucha herencia cultural amerindia, particularmente en lo referente al manejo de recursos naturales en el entorno de la llanura aluvial (Frechione et al., 1999; Parker, 1989). A los caboclos se les considera mestizos, aunque usualmente descienden directamente de los amerindios, evidenciando que el término mestizo a menudo se emplea más como una caracterización cultural que racial, para gente que ya no se identifica con una cultura étnica nativa.

La mayor parte de la población actual que vive en la llanura aluvial del Perú, habla español y se les denomina "ribereños". Igual que los caboclos del Brasil, ellos descienden de amerindios, quienes en gran medida se mezclaron con los inmigrantes y por lo tanto, también son considerados mestizos. Poco después de la llegada de los europeos, las poblaciones de la llanura aluvial del Perú fueron devastadas y diezmadas ferozmente (Santos, 1992). Más tarde, durante los siglos 16 y 17, los Jesuitas y los Franciscanos asentaron a los nativos de la región en poblados de Misioneros y fueron convertidos al catolicismo. Utilizaron la lengua quechua originaria de los Incas de la región andina, como la lengua común de la Iglesia (Stocks, 1981; Santos, 1992; San Román, 1994). Los Jesuitas fueron expulsados del Perú en 1767, pero 
desde entonces el quechua ha sido utilizado por varios grupos indígenas, especialmente a lo largo de los ríos Napo, Pastaza y Tigre. Muchas palabras quechuas relativas a los recursos naturales han sido incorporadas al actual castellano que se habla en la Amazonía.

Durante el auge del caucho, de 1880 a 1915, se aceleró el proceso de mestizaje de los nativos de la Amazonía peruana. Ingresaron nuevos inmigrantes en la región y los típicos "patrones" conocidos como los "barones del caucho", obligaron a grupos de amerindios a extraer caucho de poblaciones naturales de árboles de caucho (principalmente Hevea brasiliensis (Willdenow ex Adr. Jussieu) Mull. Arg.). Usualmente mezclaban diferentes grupos étnicos y los obligaban a desplazarse hacia áreas distantes abundantes en árboles de caucho (Collier, 1981; Pennano, 1988). Alrededor de 1915, colapsó el mercado del caucho debido a la competencia de las plantaciones de Malasia. Sin embargo, el sistema del patrón sobrevivió con los propietarios de fincas y haciendas extractivas quienes virtualmente eran dueños de los habitantes locales. Durante las décadas posteriores, la extracción de varios otros productos forestales (Padoch, 1988) estimuló migraciones distantes y el continuo mestizaje de los diversos y, de hecho, esclavizados grupos étnicos, según lo describieron Padoch $\&$ de Jong (1990) respecto a la comunidad de Santa Rosa, localizada aguas arriba de Jenaro Herrera. En Santa Rosa, los habitantes descienden de tres grupos nativos: Asháninka, Cocama y Quechua, pero hoy en día, el pueblo es una típica comunidad mestiza ribereña.

A pesar de estas irrupciones, lograron sobrevivir dos grandes culturas indígenas de la llanura aluvial del Perú: los Shipibo-Conibo de la familia lingüística Pano, y los Cocama-Cocamilla de la familia lingüística Tupí-Guaraní. Estos últimos actualmente suman 25000 habitantes aproximadamente (San Román, 1994) y viven en más de 100 comunidades, a lo largo de la parte alta y central del río Ucayali y de sus tributarios (Timoteo, 1989), que más o menos corresponde en términos geológicos al sub-valle del Ucayali (Rossanen et al., 1993). La cultura Shipibo-Conibo se distingue por sus hermosas faldas y sus famosas cerámicas. Los Cocama-Cocamilla, usualmente tienden a ocultar su identidad nativa (Stocks, 1981), por consiguiente, parecen tener pocas diferencias étnicas con los Shipibo-Conibo. Sin embargo, según San Román (1994) inclusive son más numerosos, sumando hasta 350000 habitantes. Ellos viven principalmente a lo largo de las zonas bajas de los ríos Ucayali, Marañón y Huallaga, correspondiente al sub-valle geológico Pastaza-Marañón Rässänen et al., 1993), pero también se encuentran aguas abajo a lo largo del río Amazonas, cerca de Iquitos (Villarejo, 1988). El sub-grupo Cocamilla vive a lo largo del bajo Huallaga (Stocks, 1981), en el canal del Puinahua en el río Ucayali, así como en el área del río Samiria del río Marañón; aparentemente más del 80\% de 
la población son de la etnia Cocama, mientras que el correspondiente porcentaje de la región de Jenaro Herrera, probablemente no excede del $20 \%$.

La dinámica historia demográfica y cultural de la llanura aluvial del Perú está ilustrada en el desarrollo del distrito de Jenaro Herrera. Las comunidades de Iricahua y Casa Grande (Tabla 5) se consideran pueblos amerindios Cocama, aunque sus habitantes están perdiendo su identidad nativa. Al contrario, Cedro Isla, que está ubicada en la misma isla de Casa Grande, fue fundada por inmigrantes del Departamento de San Martín. Por lo tanto, los poblados que se encuentran localizados muy cerca uno del otro, sorprendentemente son diferentes en su historia y en sus raíces étnicas según lo señalan Padoch \& de Jong (1990). La diseminación de diversos grupos religiosos en la región Amazónica del Perú, según lo describe Neyra (1992), también estimula la formación de nuevos poblados. Recientemente, el pueblo Nueva York fue establecido en 1993 por cerca de 10 familias Evangélicas Protestantes, que se desplazaron desde el pueblo Católico Cedro Isla. En 1982, el pueblo Nuevo Aucayacu fue fundado por Evangélicos Protestantes llegados del Departamento de San Martín.

Durante los últimos cien años, el número de poblados así como la población han aumentado considerablemente en el área de Jenaro Herrera (Tabla 5). Cedro Isla, Iricahua y Yanallpa eran los únicos pueblos que existían a comienzos del siglo 20 y que sólo tenían algunos cientos de habitantes. Hoy en día más de 4000 habitantes viven en 12 pueblos, y el pueblo de Jenaro Herrera, que fue fundado en 1944, para 1993 ya contaba con 2000 pobladores. La misma tendencia demográfica se observa en todas partes, a lo largo de los principales ríos donde crece el número de poblados, conservando estable el tamaño promedio de pequeños caseríos; cuando por el contrario, los pueblos grandes muestran un acelerado crecimiento poblacional que los va convirtiendo en pequeñas ciudades.

La configuración del terreno del distrito de Jenaro Herrera se compone de terrazas altas no inundables hacia el este, bordeada por la llanura aluvial hacia el Oeste. Las tres comunidades de Nuevo Aucayacu, Pumacahua y Jenaro Herrera están localizadas en el límite entre la llanura aluvial y tierra firme. La mayoría de los cultivos se realizan en tierra firme. Las 10 comunidades restantes están asentadas en la llanura aluvial donde realizan la mayor parte de sus cultivos, y de vez en cuando la gente extrae recursos de los bosques de altura (Kvist et al., en esta edición). Esta opción no la disfrutan las comunidades ubicadas aguas arriba del río Ucayali, por ejemplo las del canal de Puinahua, para quienes el área de tierra firme más cercana se encuentra a casi $80 \mathrm{~km}$. 


\subsection{La extracción y el uso económico de los hábitat de la llanura aluvial}

En la Tabla 6 se detalla la importancia de los hábitat de la llanura aluvial anteriormente identificadas (Tabla 4) para actividades extractivas y económicas. Se hace una evaluación de la importancia socioeconómica de cada actividad en todos los hábitat: principal importancia (xxx), importancia intermedia (xx) e importancia menor (x). Los puntos son subjetivamente añadidos en base a los estudios socio-económicos realizados en los pueblos de la llanura aluvial de las regiones de Jenaro Herrera, río Samiria, y el río Pacaya (Gram et al., enviada; Gram et al., en esta edición). Como un instrumento del método aplicado, el número total de cruces dentro de cada actividad no refleja su valor total socioeconómico. Los puntos se ponen cuando las actividades revisten una característica no intencionada a favor del extractor, por ejemplo, cuando los cazadores hacen largos viajes hacia distantes restingas de la llanura aluvial, y ocasionalmente matan una presa durante la travesía por una tahuampa de la llanura aluvial o por un bajial.

La Tabla 6 muestra que los hábitat ubicados en el cinturón de meandros, obtienen puntos en casi todas las actividades, mientras que los hábitat de la cuenca aluvial son menos importantes. Esto podría indicar que los recursos del cinturón de meandros son más abundantes, y que poseen un elevado potencial productivo.

A menudo se observa que existe una estrecha relación entre el tamaño de los poblados y la extensión de la restinga ubicada en los alrededores, que es el hábitat de llanura aluvial más importante para la producción de árboles frutales sensibles a la inundación, y para cultivos permanentes como el plátano y la yuca. Por otro lado, otra causa podría ser también la mayor densidad poblacional presente en el cinturón de meandros debido a la infraestructura favorable, lo cual conlleva a una intensa utilización de los recursos.

Cultivos anuales como el maíz, el arroz, frijol y diversos pepinos se desarrollan predominantemente en áreas de inundación anual ubicadas cerca de los principales ríos (Tabla 6). Diversos investigadores (Hiraoka, 1995 a, 1985 b, 1989, 1992; Bergman, 1990; Chibnik \& de Jong, 1992; Padoch \& de Jong, 1992; de Jong, 1995) hicieron una descripción de la agricultura compleja y riesgosa de la llanura aluvial del Perú. Los cultivos anuales y permanentes pueden ser destruidos por las inundaciones tempranas, altas y extensas, y el río puede llevarse los campos. En consecuencia, las familias combinan varios cultivos y trabajan diferentes hábitat y sitios para minimizar el riesgo de pérdida. Los hábitat de deposición reciente, barrizales con poca vegetación y las playas se cultivan con facilidad ya que se requiere poco esfuerzo para rozar y conservar los campos en estos lugares. Sin embargo, sólo se 
dispone de un periodo de cultivo relativamente corto, ya que existe el riesgo de una repentina subida de las aguas especialmente en estos hábitat. Los hábitat de restinga también se usan bastante para la crianza de animales, porque son fértiles y sólo se inundan por un corto periodo cada año, pero la maleza crece muy rápido en estos hábitat, lo que demanda mayor mano de obra y la aplicación de otros sistemas de cultivo como la agricultura migratoria. Las plantas perennes se cultivan más en restingas altas debido a que son sensibles a las inundaciones largas. Casi todos los agricultores de la llanura aluvial combinan esta actividad con la pesca, la caza y la extracción de productos del bosque. Esta es una forma de minimizar el impacto cuando falla la agricultura, y para generar otros ingresos fuera de la venta de la cosecha.

Aparte de la agricultura, la pesca es la actividad económica más importante para la gente que vive de los hábitat de la llanura aluvial, y la mayoría de las familias se dedica a la pesca de forma regular. Diversos investigadores describieron la interacción que existe entre las poblaciones de peces y los bosques de la llanura aluvial (Gottsberger, 1978; Goulding, 1980, 1983, 1985; Guerra, 1995; Junk et al., 1997). Durante la inundación muchas especies de peces migran hacia los bosques inundados para alimentarse, y cuando baja el nivel del agua, los peces se concentran en lagos y en ríos. La pesca se torna mejor cuando los peces migran, lo cual es estimulado por la rápida subida o descenso de los niveles de agua. Cuando el nivel del agua está bajo, la pesca es buena debido a la alta densidad de peces, que quedan atrapados en pequeños cuerpos de agua congestionados. La gente pesca en todos los hábitat de llanura aluvial que se inundan de manera significativa (Tabla 6), sin embargo, la pesca en los bosques se restringe a la época del año con elevados niveles de agua (Figura 3), aunque las cochas y depresiones lodosas que quedan en los hábitat de los bosques de bajial tienen abundantes peces durante toda la estación de nivel bajo de agua. Usualmente los pescadores realizan viajes de un día o de una semana a estos lugares, luego la pesca se conserva mediante el sistema de ahumado o salado, para luego llevar a vender en los mercados urbanos.

Para la mayoría de la gente de la llanura aluvial, la caza es solamente una actividad ocasional o rara, aunque algunos se especializan en esta profesión y venden la mayor parte de su producción como carne ahumada. Durante la inundación, los animales terrestres huyen hacia las restingas altas que no están inundadas, donde los cazadores se aprovechan de la situación matando la presa sólo con machetes (Tabla 6). Por consiguiente, la caza es una actividad estacional en la llanura aluvial. Principalmente se lleva a cabo durante los meses de altos niveles de agua (Figura 2) cuando es difícil la pesca. Los cazadores en canoas se introducen por el bosque inundado 
hacia alejadas restingas de la cuenca aluvial, donde permanecen durante varios días. También cazan en restingas ubicadas cerca de los asentamientos humanos, pero en estos lugares las especies de caza más numerosas tienden a ser depredadas o extinguidas (Bodmer, 1995; Soini et al., 1996). Durante la estación de vaciante, es muy común la caza en los hábitat de aguajales, ya que muchos animales se reúnen allí para comer fruta de palmeras.

Mientras que la caza de subsistencia a menudo depreda poblaciones de especies de caza (Bodmer, 1995), son pocas las especies de plantas que han sido depredadas por la extracción de subsistencia, por lo menos no en la región de Jenaro Herrera. La Tabla 6 divide los productos de plantas extraídas de la llanura aluvial en seis categorías según el propósito de la extracción: alimentación, construcción, técnico, leña, medicinal y comercial. Los productos que se extraen para vender han sido considerados en la última categoría, lo que implica que las restantes cinco categorías sólo comprenden productos extraídos con propósitos de subsistencia. Las categorías son idénticas y han sido definidas por Kvist et al. (en prensa) exceptuando a la leña que aparece separada de la categoría técnica. La extracción de productos para la subsistencia de los bosques de la llanura aluvial del Perú se describe muy escasamente, aunque Mejía (1988), López (1988), Kvist et al. (en prensa) han entregado alguna información.

La extracción de frutas comestibles es predominante en la categoría de alimentos, ya que a excepción del palmito de palmeras, las otras plantas del bosque se consumen raramente. Las frutas para subsistencia principalmente se extraen de las restingas del cinturón de meandro, aunque los hábitat de aguajales y pantanales también proveen frutas (Tabla 6). Las restingas proveen una diversidad de frutas mientras que los aguajales producen especialmente frutas de palmeras como la Mauritia fleuxosa y hojas tiernas de Euterpe precatoria. Kvist et al. (en prensa) describen la construcción tradicional en los pueblos de la llanura aluvial del Perú. Los materiales mayormente se extraen de los bosques del cinturón de meandro cercanos a los poblados donde se realizan las construcciones, y muy raramente de lugares más distantes. Los materiales de los bosques de restinga usualmente se utilizan más que los productos de los bosques de tahuampa (Tabla 6). Los materiales que se extraen para propósitos técnicos, por ejemplo, herramientas y artesanía, provienen de una amplia selección de formaciones boscosas. Esta categoría de usos es en sí misma muy diversa. Además, usualmente es poca la cantidad de material que se requiere para usos técnicos, lo que facilita el transporte desde áreas distantes. También las plantas medicinales son fáciles de transportar, y existe una considerable diversidad de especies para estos fines, lo que implica que las plantas medicinales también se extraen de diver- 
sas formaciones boscosas, aunque aparentemente, las restingas altas y jóvenes parecen ser las zonas más importantes. En contraste, la leña se extrae de bosques cercanos a los poblados (Tabla 6). Las restingas jóvenes son importantes debido a su contenido de la tan requerida especie Calycophyllum spruceanum y por su ubicación cercana a los caseríos en el cinturón de meandro, aunque otros hábitat de restinga y tahuampa también proveen especies de leña muy requeridas.

Los productos de plantas extraídos han sido negociados e intercambiados desde tiempos aborígenes, y la extracción comercial a gran escala empezó con la llegada de los europeos a la Amazonía. Muchos productos desde entonces fueron comercializados y ya hace décadas, inclusive siglos, que algunas especies fueron depredadas, por ejemplo, las especies Castilloa elástica Sessé, Aniba roseadora Ducke y Smilax spp. Generalmente las especies de valor comercial tienden a ser sobreexplotadas (Kvist et al., en prensa). En el área de Jenaro Herrera, los productos extraídos para fines comerciales, provienen especialmente de los hábitat de aguajal y restinga (Tabla 6), de donde principalmente se extraen frutas, palmito y madera. Los pobladores de la llanura aluvial extraen a menor escala principalmente frutas y palmito. Varios investigadores (Padoch, 1998; Peters et al., 1989a, 1989b; Peters 1990; Peters \& Hammond, 1990) han discutido sobre la extracción comercial de frutas de los bosques de la llanura aluvial del Perú. A veces la gente también extrae y comercializa un poco de madera, aunque esta actividad usualmente es realizada por gente especializada en la misma, según lo detallamos más abajo.

La FAO (1993b) declaró que la extracción comercial de madera de los bosques de la llanura aluvial del Brasil ha probado ser una "bendición" económica para las industrias forestales de la Amazonía: en 1973 abastecieron el 80\% de la madera amazónica, y el $60 \%$ en 1981. Son nombradas por su importancia las siguientes especies: Calophyllum brasiliense Cambess., Carapa guianensis Aubl., Ceiba pentandra (L) Gaertn., Hura crepitans L., Ocotea spp., Platymiscium spp., y Virola spp. Además la FAO informó que actualmente sólo se puede obtener madera en bosques de llanura aluvial ubicados en lugares muy distantes.

En un estudio de la industria maderera en la Amazonía brasileña, Ros-Tonen (1993) informa lo mismo y dice que hasta comienzos de los años setenta de 80 a $90 \%$ de la materia prima provenía de la llanura aluvial, y esta cifra bajó a $60 \%$ al final de la década, y calcula que por el año 1993 este porcentaje cayó al 15\% del total del abastecimiento de madera redonda en la región amazónica del Brasil. En el mismo período, la producción subió considerablemente hasta casi 50 millones de $\mathrm{m}^{3}$ en 1989. Las especies mencionadas como importantes son las siguientes: Carapa 
guianensis, Ceiba pentandra, Hevea guianensis Aubl. Hura crepitans, Maquira schlerophylla (Ducke) C.C. Berg (sinónimo Olmedioperebea schlerophylla Ducke ), y Virola spp. La madera de muchas de estas especies cubren la demanda de la creciente industria del Triplay, aunque la Virola surinamensis (Rol.) Warb. fue en 1988 la segunda fuente más importante de madera aserrada para exportación. RosTonen (1993), y White (1978), describieron como se lleva a cabo la extracción de madera de várzea en el Perú, mediante el trabajo de madereros que operan a menudo en distantes áreas de dominio público. Usualmente venden su producción mediante intermediarios (habilitadores), quienes frecuentemente les abastecen de las necesidades básicas para la operación en el marco del clásico sistema del patrón amazónico.

Macedo \& Anderson (1993) describieron la intensa extracción de Virola surinamensis que viene ocurriendo en enormes volúmenes $\left(243 \mathrm{~m}^{3} / \mathrm{ha}\right)$, en un pantano de agua negra de río en el estuario de la Amazonía brasileña. Sucedió que esta extracción violó tremendamente las limitaciones existentes de cortes mínimos de diámetros (45 $\mathrm{cm}$ ), y además destruyó la posible regeneración de estos lotes que en el futuro podrían ser valiosas fuentes de recursos para abastecer a la industria maderera. Los autores recomiendan mejorar y reforzar las medidas de políticas forestales adecuadas a fin de garantizar el uso sostenible de los lotes. Barros \& Uhl (1995) estudiaron la extracción de madera a lo largo del río Amazonas y del estuario en el Estado amazónico brasileño del Pará. Fueron identificados diversos patrones de extracción, transporte y procesamiento de la madera. En la várzea encontraron que el corte de los árboles y la extracción del bosque se hace en forma manual, y tradicionalmente lo realizan cuadrillas de extractores que se componen de tres hombres, quienes sacan por día básicamente unos $5 \mathrm{~m}^{3}$ de madera redonda, que usualmente lo venden a intermediarios que transportan las trozas a los aserraderos. Es muy común que estas cuadrillas reciban el pago por adelantado. El transporte se hace principalmente en balsas de troncos que son llevadas hasta pequeñas, medianas y grandes industrias de procesamiento. Los precios de los troncos de várzea baja son menores que los de tierra firme (aproximadamente la mitad). Sin embargo, los costos de transporte son considerablemente bajos: el costo de transporte en balsa llega a sólo 3\% del costo que se paga para transportar troncos en camión desde el bosque de tierra firme. En la Amazonía peruana, la extracción de madera de los bosques de la llanura aluvial aún continua proveyendo grandes cantidades de materia prima a la industria de madera comercial. La Tabla 7 muestra las cantidades totales de madera extraída y producida en 1996 en los departamentos de Loreto y Ucayali, y cuanto de esta cantidad se origina de especies de árboles que crecen en los bosques de la llanura aluvial. Cabe resaltar que no toda la madera de estas especies necesariamente proviene de los bosques de la llanura aluvial, ya que muchos también crecen en bosques de tierra 
firme. Sin embargo, en los departamentos de Loreto y Ucayali cerca del $90 \%$ y el $60 \%$ de la madera extraída provienen de especies que crecen en la llanura aluvial, lo que evidencia su importancia en la extracción de madera comercial.

Las principales especies de madera comercial que se extraen actualmente de la llanura aluvial del Perú, son las siguientes: Calycophyllum spruceanum, Calophyllum brasiliense, Cedrela odorata L., Ceiba pentandra, Hura crepitans, Maquira coriacea (Karsten) C.C. Berg, Swietenia macrophylla King, Virola spp., y diversas especies de Lauraceae y Leguminosae (ver también López \& Freitas, 1990; Freitas, 1996). Probablemente muchas otras especies son potencialmente comerciales. Usualmente la extracción se lleva a cabo mediante pequeños grupos de operadores quienes trabajan para vender sus productos a o a través de intermediarios, y también se extrae a escala mayor con operaciones planificadas. En algunos casos se utilizan aserraderos flotantes. La actual legislación forestal técnicamente está más o menos bien implementada, pero inadecuadamente reforzada en la práctica. Por ejemplo, exige la preparación de planes de manejo para otorgar grandes concesiones, los que en la práctica no son más que meras formalidades burocráticas, ya que como norma son elaborados mediante estudios de escritorio, sin ningún sustento experimental y sin ninguna preocupación por la respectiva implementación. El acceso legal a los recursos del bosque amazónico se realiza a través de concesiones de diferentes tamaños, mediante las cuales es muy común obtener fácilmente licencias de explotación de corto plazo para áreas menores que 1000 ha (FAO,1993b). Esto implica que los extractores forestales, aunque posean un permiso legal, se inclinan más a aplicar el sistema "corta y saca".

Este problema es muy latente en los bosques de llanura aluvial donde la madera se puede extraer mediante simples métodos manuales que minimizan la inversión en equipos de extracción.

\section{RECONOCIMIENTOS}

A las personas que conocimos durante el trabajo de campo en las diversas comunidades. Sin su aceptación y participación los estudios de campo hubieran sido imposibles de realizar. Al Centro de Investigaciones Jenaro Herrera (CIJH) y a su personal, que trabaja con el Instituto de Investigaciones de la Amazonía Peruana (IIAP), por haber provisto facilidades a nuestra labor, información sobre los niveles de agua en Jenaro Herrera, y por su ayuda durante el trabajo de campo en Jenaro Herrera. Al proyecto de conservación Pacaya-Samiria de la WWF-DK/AIF y a su personal por haber provisto facilidades a nuestro trabajo y por su asistencia en el trabajo de campo 
en el río Pacaya y Samiria. A la Marina de Guerra del Perú, Servicio de Hidrografía y Navegación de la Amazonía, por su amable apoyo de provisión de datos de los niveles de agua en Iquitos. Al personal de la Unidad de Forestales de la Universidad de Veterinaria y Agricultura (KVL), por su ayuda en la administración del proyecto de investigación y por sus comentarios sobre el manuscrito. Al Centro de Investigación Forestal Internacional (CIFOR) y a su personal, por su ayuda en la preparación del manuscrito y por sus comentarios sobre el mismo.

La culminación del proyecto fue posible gracias al apoyo financiero de la Agencia Danesa para el Desarrollo Internacional (DANIDA), y del proyecto para la conservación del Pacaya-Samiria que se financia y gestiona a través de la Oficina Danesa del Fondo Mundial para la Naturaleza (WWF-DK) y del Arbejdernes Internationale Forum (AIF). 


\section{BIBLIOGRAFÍA}

ANDERSON, A.B. 1990. Extraction and forest management by rural inhabitants in the Amazon estuary. In: A.B. Anderson (Editor), Alternatives to deforestation: Steps toward sustainable use of the Amazon rain forest. Columbia University Press, New York, pp. 65-85.

ARDITO, W.V. 1993. Las reducciones jesuitas de Maynas. Una experiencia misional en la Amazonía Peruana. Centro Amazónico de Antropología y Aplicación Práctica, Lima, 89 pp.

ARMSTRONG, W., BRANDLE, R., JACKSON, M.B. 1994. Mechanisms of flood tolerance in plants. Acta Botanica Neerlandica, 43 (4): 307 - 358.

AYRES, J.M. 1995. As matas de várzea do mamirauá. MCT - CNPq Sociedad Civil Mamirauá, 123 pp.

BACON, P.R. 1990. Ecology and management of swamp forests in the guianas and caribbean region. In: A.E. Lugo, M. Brinson, S. Brown (Editors), Forested Wetlands. Elsevier, Amsterdam, Oxford, New York, Tokyo, pp. 213 - 250.

CARIBBEAN REGION. IN: A.E. Lugo M. Brinson S. (Editors), Forested

BALSLEV, H., LUTEYYN, J., OLLGAARD, B., HOLM-NIELSEN, L.B. 1987. Composition and structure of adjacent unflooded and floodplain forest in Amazonian Ecuador. Opera Botanica, 92: 37 - 57.

BARROS, A.C., UHL, C. 1995. Logging along the Amazon River and estaury: patterns, problems and potential. Forest Ecology and Management, 77: 87 -105 .

BAYLEY, P.B. 1989. Aquatic environments in the Amazon basin, with an analysis of carbon sources, fish production, and yield. In: D.P. Dodge (Editor), Proceedings of the international large river symposium. Can. Spec. Publ. Aquat. Sci. pp. $399-408$.

BAYLEY, P.B., PETRERE, M. 1989. Amazon fisheries: assessment methods, current status and mangement options. In: D.P. Dodge (Editor), Proceedings of the international large river symposium. Can. Spec. Publ. Fish. Aquat. Sci. pp. $385-398$. 
BERGMAN, R. 1990. Economía amazónica. Estrategias de subsistencia en las riberas del Ucayali en el Perú. Centro Amazónico de Antropología y Aplicación Práctica, Lima, 209 pp.

BIEDMA, M. 1989. La conquista franciscana del alto Ucayali. IIAP/CETA, Iquitos, $292 \mathrm{pp}$

BLACK, G,A., DOBZHANSKY, T., PAVAN, C. 1950. Some attempts to estimate species diversity and population density of trees in Amazonian forests. Botanical Gazette, 111: 413 - 425 .

BODMER, R.E. 1995. Priorities for the conservation of mammals in the Peruvian Amazon. Oryx, 29 (1): 23-28.

BRAGA, P.I.S. 1979. Subdivisao fitogeografico, tipos de vegetação, consevação e inventário floristico de floresta amazonica. Acta Amazonía, 9(4): 53-80

CAMPBELL, D.G., DOUGLAS, C.D., PRANCE, G.T., MACIEL, U.N. 1986. Quantitative ecological inventory of terra firme and varzea tropical forest on the Rio Xingu, Brazilian Amazon. Brittonia, 38(4): 369 - 393.

CHIBNIK, M., DE JONG, W. 1992. Organización de la mano de la obra agrícola en las comunidades ribereñas de la Amazonía Peruana. Amazonía Peruana, 11(21): $181-215$.

CLAUSSI, A., MARMILLOD, D., BLASER, J. 1992. Descripción silvicultural de las plantaciones forestales de Jenaro Herrera. Instituto de Investigaciones de la Amazonía Peruana, Iquitos, 334 pp.

COLLIER, R. 1981. Jague al Batón. La historia del caucho en la Amazonía. Centro Amazónica de Antropología y Aplicación Práctica, Lima, 259 pp.

COLONNELLO, G. 1990. A Venezuelan floodplain study on the Orinoco River. Forest Ecology and Management, 33/34: 103 - 124.

CRAWFORD, R.M.M. 1982. Physiological responses to flooding. Enc Plant Physiol, 12B: $453-477$.

DE JONG, W. 1995. Diversity, variation, and change in Ribereno agriculture and agroforestry. CIP-DATA Koninklijke Biliotheek, Den Haag, 168 pp. 
DENEVAN, W.M. 1976. The aboriginal population of Amazonía. In: W.M. Denevan (Editor), The native population of the Americas in 1492. The University of Wisconsin Press, pp. 205-234.

DOUROJEANNI, M.J. 1990. Amazonía. ¿Qué hacer? Centro de Estudios Teológicos de la Amazonía, Iquitos, 444 pp.

DUMONT, J.F., GARCIA, F. 1992. Hundimientos activos controlados por estructuras del basamiento en la cuenca Marañón (Noreste del Perú). Folia Amazónica, 4(1): 7 - 17.

DUMONT, J.F., LAMOTTE, S., KAHN, F. 1990. Wetland and upland forest ecosystems in Peruvian Amazonía: Plant species diversity in the light of some geological and botanical evidence. Forest Ecology and Management, 33/34: 125 - 139 .

EDEN, J.E. 1990. Ecology and land management in Amazonía. Belhaven Press, London, 269 pp.

ENCARNACIÓN, F. 1985. Introducción a la flora y vegetación de la Amazonía peruana: Estado actual de los estudios, medio natural y ensayo de una clave de determinación de las formaciones vegetales en la llanura amazónica. Candollea, 40: 237 - 252.

ENCARNACIÓN, F. 1993. El bosque y las formaciones vegetales en la llanura amazónica del Perú. Alma Máter, 6: 95 - 114.

FAO, 1993 a. Conservation of genetic resources in tropical forest management. Principles and concepts. FAO, rome. FAO Forestry Paper. No. 107. 105 pp.

FAO, 1993 b. Management and coservation of closed forests in tropical America. FAO, Rome. FAO Forestry Papers. No. 101. 141 pp.

FOSTER, R.B. 1990 a. Long-term change in the successional forest community of the Rio Manu floodplain. In: A.H. Gentry (Editor), Four neotropical rainforests. Yale University Press, New Haven and London, pp. 565 - 572.

FOSTER, R.B. 1990 b. The floristic composition of the Rio Manu Floodplain forest. In: A.H. Gentry (Editor), Four neotropical rainforests. Yale University Press, New Haven, pp. 99 - 111. 
FOSTER, R.B., ARCE, J.B., WACHTER, T.S. 1986. Dispersal and the sequential plant communities in Amazonian Peru floodplain. In: A. Estrada, T.H. Fleming (Editors), Frugivores and seed dispersal. Dr W. Junk Publishers, Dordrecht, pp. $357-370$.

FRECHIONE, J., POSEY, D.A., DA SILVA, L.F. 1989. The perception of ecological zones and natural resources in the Brazilian Amazon: An ethnoecology of Lake Coari. Advances in Economic Botany, 7: 260 - 282.

FREITAS, L.A. 1996. Caracterización florística y estructural de cuatro comunidades boscosas de la llanura aluvial inundable en la zona Jenaro Herrera, Amazonía Peruana. Instituto de Investigación de la Amazonía Peruana, Iquitos. Documento Técnico. No. 21. 73 pp.

FURCH, K. 1997. Chemistry of várzea and igapó soils and nutrient inventory of their floodplain forests. In: W.J. Junk (Editor), The central Amazon floodplain. Ecology of a pulsing system. Springer-Verlag, Berlin, pp. 47-68.

GENTRY, A.H. 1988. Tree species richness of upper Amazonian forests. Proc Natl Acad Sci USA, 85: 156-159.

GENTRY, A.H., LOPÉZ, J.P. 1980. Deforestation and increased flooding of the upper Amazon. Science, 210: 1354-1356.

GENTRY, A.H., ORTIZ, R. 1993. Patrones de composición florística en la Amazonía Peruana. In: R. Kalliola, M. Puhakka, W. Danjoy (Editors), Amazonía peruana. Vegetación húmeda en el llano subandino. PAUT/ONERN, Jyväskylä, pp. 155-166.

GENTRY, A.H., TERBORGH, J. 1990. Composition and dynamcis of the Cocha "mature" floodplain forest. In: A.H. Gentry (Editor), Four neotropica rainforests. Yale University Press, New Haven and London, pp. 542-563.

GILL, C.J. 1970. The flooding tolerance of woody species - a review. Forestry Abstracts, 31 (4) : $671-688$.

GOTTSBERGER, G. 1978. Seed dispersal by fish in the inundated regions of Huamaita, Amazonía. Biotropica, 10(3): 170-183.

GOULDING, M. 1980. The fishes and the forest. Explorations history. University of California Press, Bekerly, 280 pp. 
GOULDING, M. 1983. The role of fishes in seed dispersal and plant distribution in Amazonian floodplain ecosystems. Sonderd naturwiss Ver Hamburg, 7: $271-283$.

GOULDING, M. 1985. Forest fishes of the Amazon. In: G.T. Prance, T.E. Lovejoy (Editors), Amazonía. Pergamon Press, Oxford, pp. 267 - 276.

GOULDING, M., SMITH, N.J.H., MAHAR, D.J. 1996. Floods of fortune. Ecology and economy along the Amazon. Columbia University Press, New York, $193 \mathrm{pp}$.

GREIG - SMITH, P. 1983. Quantitative plant ecology. Blackwell Scientific Publications, $359 \mathrm{pp}$.

GRENAND, F., GRENAND, P. 1993. Histoire du peuplement de la varzea en Amazonas. Amazoniana, 12(3/4): 509 - 526.

GUERRA, H.F. 1995. Estado actual del conocimiento de la pesquería en la Amazonía Peruana. Instituto de Investigaciones de la Amazonía peruana, Iquitos. Documento Técnico. No. 11. 53 pp.

HEMMING, J. 1987. Amazon frontier. The defeat of the Brazilian Indians. Harvard University Press, Cambridge, 647 pp.

HIRAOKA, M. 1985 a. Mestizo subsistence in riparian Amazonía. National Geographic Research, 1(2): 236 - 246.

HIRAOKA, M. 1985b. Cash cropping, wage labor, and urbanward migrations: changing floodplain subsistence in the Peruvian Amazon. Studies in Third World Countries, 32: 199-243.

HIRAOKA, M. 1989. Riberenos' changing economic patterns in the Peruvian Amazon. Journal of Cultural Geography, 9(2): 103-119.

HIRAOKA, M. 1992. Caboclo and ribereno resource management in Amazonía: A review. In: K.H. Redford, C. Padoch (Editors), conservation of Neotropical forests. Working from traditional resource use. Columbia University Press, New York, pp. 134-157.

HOOK, D.D. 1984. Adaptations to flooding with flesh water. In: T.T. Kozlowski (Editor), flooding and plant growth. Academic Press, pp. 265-294. 
IIAP, 1997. Zonificación de los bosques de producción de la región de Loreto. IIAP, Iquitos, $30 \mathrm{pp}$.

INEI, 1997. Instituto Nacional de Estadística e Informática. http://161.132.90.5/ bancogeo, 29-12-97.

IRION, G., JUNK, W.J., DE MELLO, J.A.S.N. 1997. The large central amazonian river floodplain near Manaus: geological, climatological, hidrological, and geomorphological aspects. In: W.J. Junk (Editor), The central Amazon floodplain. Ecology of a pulsing system. Springer-Verlag, Berlin, pp. 23-46.

JUNK, W.J. 1983. Ecology of swamps on the middle Amazon. In: A.J.P. Gore (Editor), Mires: swamp, borg, fen and moor. Elsevier, Amsterdam, pp. 269294.

JUNK, W.J. 1984. Ecology of the várzea, floodplain of Amazon white-water rivers. In: H. Sioli (Editor), The Amazon. Limnology and landscape ecology of a mighty river and its basin. Dr W. Junk Publishers, Dodrecht, pp. 213-243.

JUNK, W.J. 1989. Flood tolerance and tree distribution in central Amazonian floodplains. In: L.B. Holm-Nielsen, I.C. Nielsen, H, Balslev, H. Balslev (Editor), tropical forests. Botanical dynamics, speciation and diversity. Academic Press Limited, pp. 47-64.

JUNK, W.J. 1993. Wetlands of tropical South America. In: D. Whigham, D. Dykyjova, S. Hejny (Editors), Wetlands of the world: inventory, ecology and management. Kluwer Academic Publisher, Dordrecht, pp. 679-739.

JUNK, W.J. 1997. General aspects of floodplain ecology with special reference to Amazonian floodplains. In: W.J.

JUNK(EDITOR), The central Amazon floodplain. Ecology of a pulsing system. Springer- Verlag, Berlin, pp. 3-20.

JUNK, W.J., BAYLEY, P.B. SPARKS, R.E. 1989. The flood pulse concept in riverfloodplain systems. In: D.P. Dodge (Editor), Proceedings of the international large river symposium. Can. Spec. Publ. Fish. Aquat. Sci. pp. 110-127.

JUNK, W.J., DA SILVA, V.M.F. 1997. Mammals, Reptiles and Amphibians. In: W.J. Junk (Editor), The central Amazon floodplain. Ecology of a pulsing system. Springer-Verlag, Berlin, pp. 409-417. 
JUNK, W.J., PIEDADE, M.T.F. 1993. Herbaceous plants of the Amazon floodplain near Manaus: Species diversity and adaptations to the flood pulse. Amazoniana, 12(3/3): 467-484.

JUNK, W.J., PIEDADE, M.T.F. 1997. Plant life in the floodplain with special reference to herbaceous plant. In: W.J.

JUNK (Editor), The central Amazon floodplain. Ecology of a pulsing system. Springer-Verlag, Berlin, pp. 147-185.

JUNK, W.J., SOARES, M.G.M., SAINT-PAUL, U. 1997. The fish. In: W.J. Junk (Editor), The central Amazon floodplain. Ecology of aaa pulsing system. Springer-Verlag, Berlin,pp. 385-408.

KAHN, F., MEJIA, K. 1990. Palm communities in wetland forest ecosystems of Peruvian Amazonía. Forest Ecology and Management, 33/34: 169-179.

KALLIOTA, R., PUHAKKA, M. 1993. Geografía de la selva baja peruana. In: R. Kalliola, M. Puhakka,W. Danjoy (Editor), Amazonía Peruana. Vegetación húmeda tropical en el llano subandino. PAUT/ONERN, Jyväskylä, pp. 9-22.

KALLIOTA, R., PUHAKKA, M., SALO, J., TUOMISTO, H., RUOKOLAINEN, K. 1991 a. The dynamics, distribution and classification of swamp vegetation in Peruvian Amazonía. Ann Bot Fennici, 28: 225-239.

KALliOTA, R., SALO, J., PUHAKKA, M., RAJASILTA, M. 1991b. New site formation and colonizing vegetation in primary succession on the western Amaazon floodplains. Journal of Ecology, 79: 877-901.

KALLIOTA, R., SALO, J., PUHAKKA, M., RAJASILTA, M., NELLER, R., RASANEN, M.E., DANJOY, W.A.A. 1992. Upper Aamazon channel migration . Naturwissenshaften, 79: 75-79.

KEEL, S.H.K., PRANCE, G.T. 1979. Studies of the vegetation of a white-sand blackwater igapo (Rio Negro, Brazil). Acta Amazonica, 9 (4): 645-655.

KLINGE, H., JUNK, W.J., REVILLA, C.J. 1990. Status and distribution of forested wetlands in tropical South America. Forest Ecology and Management, 33/ 34: 81-101. 
KOZLOWSKI, T.T. 1984. Responses of woody plants to flooding. In: T.T. Kozlowski (Editor), Flooding and plant growth. Academic Press, pp. 129-163.

KUBITZKI, K. 1989. The ecogeographical differentaation of Amazonian inundation forets. P1 Syst Evol, 162: 285-304.

KUBITZKI, K. ZIBURSKI, A. 1994. Seed dispersal in flood plain forests of Amazonía. Biotropica, 26(1): 30-43.

KVIST, L.P., GRAM, S., CACARES A.C., ORÉ, I.B. 1999 a. Socio-economy of villagers in the Peruvian Amazon With a particular focus at extraction: A comparison of seven flood plain communities along the lower Ucayali and Marañon rivers. Royal Veterinary and Agricultural University, Department of Economics and Natural Resources, Unit of Forestry. Copenhagen. Unpublished manuscript.

KVIST, L.P., ANDERSEN, M.K., STAGEGAAARD, J. HESSELSOE, M., LLAPAPASCA, C. 1999b. Extraction from woody forest plants in flood plain communities in Amazonian Peru: Evaluation, choice and conservation status of resources. Royal Veterinary and Agricultural University, Department of Economics and Natural Resources, Unit of Forestry. Copenhagen. Unpublished maanuscript.

LAMOTTE, S. 1990. Fluvial dynamics and succession in the Lower Ucayali River basin, Peruvian Amazonía. Forest Ecology and Management, 33/34: 141156.

LONZOY, A.A. 1992. De nativos a ribereños un recorrido a lo largo de su historia comunal. Servicio Holandés de Cooperación Técnica y Social (SNV), Iquitos, $163 \mathrm{pp}$.

LÓPEZ, J.P. 1988. The use of palms and other native plants in non-convetional, low cost rural housing in the Peruvian Amazon. Advances in Economic Botany, 6: 119-129.

LÓPEZ, J.P. FREITAS, D. 1990. Geographical aspects of forested wetlands in the Lower Ucayali, Peruvian Amazonía. Forest Ecology and Management, 33/ 34: $157-168$.

LUGO, A.E., BROWN., S., BRINSON, M.M. 1990 a. Concepts in wetland ecology. In: A.E. Lugo, M Brinson, S. Brown (Editor), Forested wetlands. Elsevier, Amsterdam, pp. 53-85. 
LUGO, A.E., BRINSON, M.M., BROWN, S. 1990b. Synthesis and search for paradigms in wetland ecology. In: A.E. Lugo, M. Brinson, S. Brown (Editor), Forested wetlands. Elsevier, Amsterdam, pp. 447-460.

MACEDO, D.S., ANDERSON, A.B. 1993. Early ecological changes associated with logging in a Amazonian floodplain. Biotropica, 25(2): 151-163.

MEGGERS, B.J. 1971. Amazonía. Man and culture in a counterfeit paradise. AHM Publishing Corpotation, Illinois, $182 \mathrm{pp}$.

MEJÍA, K. 1988. Utilization of palms in eleven mestizo villages of the Peruvian Amazon (Ucyali river, Department of Loreto). Advances in Economic Botany, 6: 130-136.

MORAN, E.F. 1989. Models of native and folk adaptation in the Amazon. Advances in Economic Botany, 7: 22-29.

MORAN, E.F. 1993. La ecología humana de los pueblos de la Amazonía. Fondo de Cultura Económica, México, 325 pp.

NAMKOONG, G., Boyle, T., Gregorius, H., Joly, H., Savolainen, O., Ratnam, W., Young, A. 1996. Testing criteria and indicators for assessing the sustainability of forest management: Genetic criteria and indicators. CIFOR, Bogor. CIFOR Working Paper. no. 10.

NEBEL, G., KVIST, L. P., VANCLAY, J. K., CHRISTENSEN, H., FREITAS, L., RUÍZ, J. 1999 a. Structure and floristic composition of flood plain forests in the Peruvian Amazon: I. Overstorey. Royal Veterinary and Agricultural University, Department of Economics and Natural Resources, Unit of Forestry. Copenhagen. Unpublished manuscript.

NEBEL, G., DRAGSTED, J., VANCLAY, J.K. 1999 b. Structure and floristic composition of flood plain forests in the Peruvian Amazon: II. The understorey of restinga forests. Royal Veterinary and Agricultural University, Department of Economics and Natural Resources, Unit of Forestry. Copenhagen. Unpublished manuscript.

NEYRA, J. G. 1992. Los que llegaron después... Estudios del impacto cultural de las denominaciones religiosas no católicas en Iquitos. CETA, Iquitos, 381 pp. 
NORDIN, C. F., MEADE, R. H. 1982. Deforestation and increased flooding of the Upper Amazon. Science, 215: 426 - 427.

OHLY, J. J. 1985. Die Wasserbuffelwirtschaft am mittleren Amazonas aus der sicht der jungsten Entwicklungen. Amazoniana, 9(3): 441 - 457.

PADOCH, C. 1988. People of the floodplain and forest. In: J.S. Denslow, C. Padoch (Editors), People of the tropical rain forest. University of California Press, Berkeley, pp. $127-140$.

PADOCH, C., DE JONG, W. 1990. Santa Rosa: The impact of the forest products trade on an Amazonian place and population. Advances in Economic Botany, 8: $151-158$.

PADOCH, C., DE JONG, W. 1992. Diversity, variation, and change in ribereño agriculture. In: K. H. Redford, C. Padoch (Editors), Conservation of neotropical forests. Working from traditional resource use. Columbia University Press, New York, pp. 158 - 174.

PARKER, E.P. 1989. A neglected human resource in Amazonía: The Amazon caboclo. Advances in Economic Botany, 7: 249-259.

PARSSINEN, M.H., SALO, J., RÄSSÄNEN, M.E. 1996. River floodplain relocations and the abandonment of aborigine settlements in the Upper Amazon Basin: a historical case estudy of San Miguel de Cunibos at the Middle Ucyali river. Geoarchaelogy, 11(4): 345-359.

PENNANO, G. 1988. La economía del caucho. CETA, Iquitos, 264 pp.

PETERMANN, P. 1997. The birds. In: W.J. Junk (Editor), The Central Amazon floodplain. Ecology of a pulsing system. Springer - Verlang, Berlin, pp. 419 - 452.

PETERS, C. M. 1990. Population ecology and management of forest fruit trees in Peruvian Amazonía. In: A.B. Anderson (Editor), alternatives to deforestation: Steps toward sustainable use of Amazon rain forest. Columbia University Press, New York, pp. 86-98.

PETERS, C.M., BALICK, M.J., KAHN, F., ANDERSON, A.B. 1989a. Oligarchic forests of economic plants in Amazonía: Utilization and conservation of an important tropical resource. Conservation Biology, 3(4):341-349. 
PETERS, C.M., GENTRY, A.H., MENDELSON, R.O. 1989b. Valuation of an Amazonía rainforest. Nature, 339:655-656.

PETERS, C.M., HAMMOND, E.J. 1990. Fruits from the flooded forests of Peruvian Amazonía: Yield estimates for natural populations of three promimising species. Advance in Economic Botany, 8:159-176.

PIRES, J.M., PRANCE, G.T. 1985. The vegetation types of the Brazilian Amazon. In: G.T. Prance, T.E. Lovejoy (Editor), Amazonía. Pergamon Press, Oxford, pp. 109-145.

PRANCE, G.T. 1979. Notes on the vegetation of Amazonía III. The terminology of Amazonian forest types subject to inundation. Brittonia, 31(1):26-38.

PRANCE, G.T. 1980. A terminologia dos tipos de floresta amazonicas sujeitas a inundaçao. Acta Amazonica, 10(3):495-504.

PUHAKKA, M., KALLIOLA, R. 1993. La vegetación en áreas de inundación en la selva baja de la Amazonía Peruana. In: R. Kalliola, M. Puhakka, W. Danjoy (Editors), Amazonía peruana. Vegetación húmeda tropical en el llano subandino. PAUT/ONERN, Jyväskylä, pp. 113-138.

PUHAKKA, M., KALLIOLA, R., RAJASITA, M. SALO, J. 1992 River types, site evolution and successioanal vegetation patterns in Peruvian Amazonía. Journal of Biogeography, 19: 651-665.

PUHAKKA, M., KALLIOLA, R., SALO, J., RAJASITA, M. 1993. La sucesión forestal que sigue a la migración de ríos en la selva baja peruana. In: R. Kalliola, M. Puhakka, W. Danjoy (Editors), Amazonía peruana. Vegetación húmeda tropical en el llano subandino. PAUT/ONERN, Jyväskylä, pp. 167-201.

RÄSÄNEN, M. 1993. La geohistoria de vegetación en la selva baja peruana. In: R. Kalliola, M. Puhakka, W. Danjoy (Editors), Amazonía peruana. Vegetación húmeda tropical en el llano subandino. PAUT/ONERN, Jyväskylä, pp. 43-68.

RÄSÄNEN, M., KALLIOLA, R., PUHAKKA, M. 1993. Mapa geoecológico de la selva baja peruana: explicaciones. In: R. Kalliola, M. Puhakka, W. Danjoy (Editors), Amazonía peruana. Vegetación húmeda tropical en el llano subandino. PAUT/ONERN, Jyväskylä, pp. 207-216. 
RÄSÄNEN, M.E., NELLER, R., SALO, J., JUNGNER, H. 1992. Recent and ancient fluvial deposition systems in the Amazonian foreland basin, Peru. Geol Mag, 129(3): 293-306.

RÄSÄNEN, M.E., SALO, J., JUNGNER, H., PITTMAN, L.R. 1990. Evolution of the Western Amazon lowland relief: impact of Andean foreland dynamics. Terra Nova, 2: 320-332

RÄSÄNEN, M.E., SALO, J., KALLIOLA, R. 1987. Fluvial perturbance in the Western Amazon basin: regulation by long-term sub-Andean tectonics. Science, 238: 1398-1401.

REINECK, H.E., SINGH, I.B. 1980. Depositional sedimentary environments. Springer-Verlag, Berlin, $551 \mathrm{pp}$.

RODRÍGUEZ, A. F., RODRÍGUEZ, A.M., VÁSQUEZ, R.P. 1995. Realidad y perspectiva. La Reserva Nacional Pacaya-Samiria. Pro Naturaleza, Lima, 132 pp.

ROOSEVELT, A. 1989. Resource management in Amazonía before the conquest: Beyond ethnographic projection. Advances in Economic Botany, 7: 30-62.

ROS-TONEN, M.A.F. 1993. Tropical hardwood from the Brazilian Amazon. Verlang Breitenbach Publishers, Saarbrücken - Fort Lauderdale, 279 pp.

ROSS, E.B. 1978. The evolution of the Amazon Peasantry. J Lat Amer Stud, 10(2): 193-218

SALATI, E. 1985. The climatology and hydrology of Amazonía. In: G.T. Prance, T.E. Lovejoy (Editors), Amazonía. Pergamon Press, Oxford, pp. 18-49.

SALO, J., KALLIOLA, R. 1993. Necesidades para el futuro en la selva baja peruana: Conservación e investigación científica. In: R. Kalliola, M. Puhakka, W. Danjoy (Editors), Amazonía peruana. Vegetación húmeda tropical en el llano subandino. PAUT/ONERN, Jyväskylä, pp. 235-250.

SALO, J., KALLIOLA, R. HÄKKINEN, I., MÄKINEN, Y., NIEMELÄ, P., PUHAKKA, M., COLEY, P.D. 1986. River dynamics and the diversity of Amazon lowland forest. Nature, 322: 254-258.

SALO, J., RÄSÄNEN, M.E. 1989. Hierarchy of landscape patterns in western Amazon. In: L.B. Holm-Nielsen, I.C. Nielsen, H. Balslev (Editors), Tropi- 
cal forests. Botanical dynamics, speciation and diversity. Academic Press, pp. 239-251.

SAN ROMÁN, J. 1994. Perfiles históricos de la Amazonía peruana. CETA/CAAAP/ IIAP, Iquitos, $274 \mathrm{pp}$.

SANTOS, F. 1992. Etnohistoria de la alta Amazonía. Siglos XV-XVIII. ABYA/ YALA, Quito, $305 \mathrm{pp}$.

SCHLUTER, U.B., FURCH, V.B., JOLY, C.A. 1993. Physiological and anatomical adaptation by young Astrocaryum jauari Mart. (Arecaceae) in periodically inundadted biotopes of Central Amazonía. Biotropica, 25(4): 384-396.

SCHMIDT, G.W., 1972. Amounts of suspended solids and dissolved substances in the middle reaches of the Amazon over the course of one year (August, 1969-July, 1970). Amazoniana, 3(2): 208-223.

SEIDENSCHWARZ, F. 1986. Vergleich von Flussuferkrautgesselschaften mit Wildkrautvegetation im tropischen Tiefland von Peru. Amazoniana, 10(1): 79-111.

SIOLI, H. 1968. Hydrochemistry and geology in the Brazilian Amazon region. Amazoniana, 1(3): 267-277.

SIOLI, H. 1984. The Amazon and its main afluents: Hydrography, morphology of the river courses, and river types. In: H. Sioli (Editor), The Amazon. Limnology and landscape ecology of a mighty tropical river and its basin. Dr. W. Junk Publishers, Dodrecht, pp. 127-165.

SIOLI, P., SICCHAR, L.A., GIL, N.G., FACHIN, T.A., PEZO, R., CHUMBE, A.M. 1996. Una evaluación de la fauna silvestre y su aprovechamiento de la Reserva Nacional Pacaya-samiria, Peru. Instituto de Investigaciones de la Amazonía Peruana, Iquitos. Documento Técnico. No. 24. 64 pp.

STOCKS, A.W. 1981. Los nativos invisibles. Centro Amazónico de Antropología y Aplicación Práctica, Lima, 185 pp.

SORENSEN, M., DOYGAARD, S., ESTRELLA, J.E., KVIST, L.P., NIELSEN, P.E. 1997. Status of the South American tuberous legume Pachyrhizus tuberosis (Lam) Spreng. Biodiversity and Conservation, 6: 1581-1625. 
TAKEUCHI, M. 1962. The structure of Amazonian vegetation. VI. Igapó. Journ Fac Sci Univ Tokyo, 8(7): 297-308.

TIMOTEO, C. 1989. Los Unaya y su mundo. Aproximación al sistema médico de los Shipibo-Conibo del Río Ucayali. Centro Amazónico de Antropología y Aplicación Práctica, Lima, 291 pp.

TUOMISTO, H. 1993. Clasificación de vegetación en la selva baja peruana. In: R. Kalliota, M. Puhakka, W. Danjoy (Editor), Amazonía peruana. Vegetación humeda en el llano subandino. PAUT/ ONERN, Jyväskylä, pp. 103-112.

VILLAREJO, A 1988. Así es la selva. CETA, Iquitos, 330 pp.

WALKER, I. 1995. Sedimentacion in the inundation forest Flanking the Central Amazonian blackwater stream Rio Tarauma Mirin (Manaus, Amazonas State). Amazoniana, 13 (3/4): 237-243.

WELCOMME, R.L. 1985. River fisheries. FAO, Rome.FAO Fisheries Technical Paper. no. 262. 326 pp.

WHITE, S. 1978. Cedar and mahogany logging in eastern Peru. The Geographical Review, 68: 394-416.

WORBES, M. 1983. Vegetationskundliche Untersuchungen zweier Uberschwemmungswalder in Zentralamazonien - vorlaufige Ergebnisse. Amazoniana, 8(1): 47-65.

WORBES, M. 1986. Lebensbedingungen und Holzwachstum in Zentralamazonischen Uberschwemmungswaldern. Scripta Geobotanica, 17: 7-112.

WORBES, M. 1997. The Forest ecosystem of the floodplains. In: W.J. Junk (Editor), The central Amazon floodplain. Ecology of a pulsing system. Springer - Verlag, Berlin, pp. 223-266.

WORBES, M. KLINGE, H. KLINGE, H., REVILLA, J.D. MARTIUS, C. 1992. On the dynamic, floristic subdivision and geographical distribution of varzea forests in Central Amazonía. Journal of Vegetation Science, 3: 553-564.

ZIBURSKI, A. 1991. Dissemination, Keimung und atablierung einiger baumarten der uberschwemmungswalder Amazoniens. Tropische und Subtropische Pflanzenwelt, 77: 1-96. 
BOSQUE DE LA LLANURA ALUVIAL DEL PERÚ: ECOSISTEMAS,

Tabla 1. Recursos de fauna en la llanura aluvial del perú presentes en la región baja del río Ucayali y río Merañon. Las especies seleccionadas son aquéllas que reciben influencia negativa de las actividades humanas. Basado en Soini et al. (1996) .

\begin{tabular}{|c|c|c|c|c|}
\hline Nombre científico & Nombre local & Nombre inglés & Uso/problemas & Estado \\
\hline \multicolumn{5}{|l|}{ PECES } \\
\hline Arapaima gigas & Paiche & Arapaima & Alim, mascota & Explotado \\
\hline Osteoglossum bicirrhosum & Arawana & Aruaña & Alim, mascota & Localmente explot \\
\hline \multicolumn{5}{|l|}{ REPTILES } \\
\hline Melanosuchus niger & Lagarto negro & Black caiman & Cuero, dañino & Explotado \\
\hline Caiman crocodylus & Lagarto blanca & Spectacled caiman & Alim, dañino & Localmente explot \\
\hline Geochelone denticulata & Motelo & & Alimento & Localmente explot \\
\hline Podocnemis sextuberculata & Cupiso & & Alimento & Localmente explot \\
\hline Podocnemis unfilis & Taricaya & Big headed turtle & Alimento & Explotado \\
\hline Podocnemis expansa & Charapa & Giant Amazon river turtle & Alimento & En peligro \\
\hline \multicolumn{5}{|l|}{ PÁJAROS } \\
\hline Ara macao & Guacamayo rojo & Scarlet macaw & Alim, mascota & Explotado \\
\hline Crax mitu & Paujil & Razor-billed curassow & Alimento & Localmente explot \\
\hline Cairina moschata & Sacha pato & Muscovy duck & Alimento & Localmente explot \\
\hline \multicolumn{5}{|l|}{ MAMÍFEROS } \\
\hline Myrmecophaga tridactyla & Oso hormiguero & Giant anteater & Alimento & Explotado \\
\hline Priodontes maximus & Yungunturo & Giant armadillo & Alimento & Explotado \\
\hline Lagothrix lagothricha & Choro & Common woolly monkey & Alimento & Explotado \\
\hline Alouatta seniculus & Coto & Red howler monkey & Alimento & Localmente explot \\
\hline Cacajoa calvus rubicundus & Huapo colorado & Red uacari monkey & Alimento & En peligro \\
\hline Pithecia monachus & Huapo negro & Mon saki monkey & Alimento & Explotado \\
\hline Ateles belzebuth & Maquisapa ceniza & White-bellied spider monkey & Alimento & Explotado \\
\hline Ateles paniscus & Maquisapa negro & Black spider monkey & Alimento & En peligro \\
\hline Cebus apella & Mono negro & Brown capuchin monkey & Alimento & En peligro \\
\hline Cebus albifrons & Mono blanco & White-fronted capuchin & Alimento & Localmente explot \\
\hline Felis pardalis & Tigrillo & Ocelot & Piel, nocivo & Localmente explot \\
\hline Panthera onca & Otorongo & Jaguar & Piel, nocivo & Explotado \\
\hline Lutra longicaudis & Nutria & Southern river otter & Piel, alimento & Localmente explot \\
\hline Pteronura brasiliensis & Lobo del rio & Giant otter & Piel & En peligro \\
\hline Trichechus inunguis & Vaca marina & Manatee & Alimento & En peligro \\
\hline Tapirus terrestris & Sacha vaca & Brazilian tapir & Alimento & Localmente explot \\
\hline
\end{tabular}


Tabla 2. Recursos de plantas de la llanura aluvial del perú presentes en la región baja del río Ucayali y río Marañón , y sus usos más importantes. Las especies que se utilizan para fines de subsistencia, principalmente son depredadas cerca de los poblados (locales), mientras que las especies que se comercializan, generalmente son más depre dadas.

\begin{tabular}{|c|c|c|c|c|}
\hline Nombre científico & Familia & Nombre local & Uso principal & Estado \\
\hline $\begin{array}{l}\text { Aniba spp., Ocotea spp. } \\
\text { Brunfelsia grandiflora D. Don } \\
\text { Calophyllum brasiliensis } \text { Cambess } \\
\text { Castilla } \text { ulei } \text { Warb } \\
\text { Cedrela odorata } \text { L. } \\
\text { Ceiba pentandra } \text { (L.) Gaertn. } \\
\text { Euterpe precatoria } \text { Mart. } \\
\text { Ficus insipida Willd. } \\
\text { Mauritia flexuosa } 1 . \text { F. } \\
\text { Maytenus macrocarpa } \text { (Ruiz \& } \\
\text { Pav.) Briq. } \\
\text { Minquartia guianensis Aubl. } \\
\text { Phytelephas macrocarpa } \text { Ruiz \& } \\
\text { Pav. } \\
\text { Schelea } \text { spp. } \\
\text { Smilax } \text { sp. } \\
\text { Swietenia macrophylla } \text { King } \\
\text { Uncaria } \text { spp. } \\
\text { Virola } \text { spp. }\end{array}$ & $\begin{array}{l}\text { Lauraceae } \\
\text { Solanaceae } \\
\text { Clusiaceae } \\
\text { Moraceae } \\
\text { Meliaceae } \\
\text { Bombacaceae } \\
\text { Arecaceae } \\
\text { Moraceae } \\
\text { Arecaceae } \\
\text { Celastraceae } \\
\text { Olacaceae } \\
\text { Arecaceae } \\
\text { Arecaceae } \\
\text { Smilacaceae } \\
\text { Meliaceae } \\
\text { Rubiaceae } \\
\text { Myristicaceae }\end{array}$ & $\begin{array}{l}\text { Moena } \\
\text { Chiric sanango } \\
\text { Alfaro, Lagarto caspi } \\
\text { Caucho } \\
\text { Cedro } \\
\text { Lupuna } \\
\text { Huasai } \\
\text { Ojé } \\
\text { Aguaje } \\
\text { Chuchuhuasi } \\
\\
\text { Huacapú } \\
\text { Yarina } \\
\\
\text { Shapaja } \\
\text { Zarzaparilla } \\
\text { Aguano, Caoba } \\
\text { Uña de gato } \\
\text { Cumala, Cumala } \\
\text { caupuri, Aguano } \\
\text { cumala }\end{array}$ & $\begin{array}{l}\text { Madera aserrada } \\
\text { Raíz para medicina } \\
\text { Madera } \\
\text { Resina } \\
\text { Madera aserrada } \\
\text { Tryplay } \\
\text { Palmito comestible } \\
\text { Resina } \\
\text { Frutos } \\
\text { Corteza para medicina } \\
\text { Maderaje para construcción } \\
\text { Hojas para techo } \\
\\
\text { Hojas para techo } \\
\text { Raíz para medicina } \\
\text { Madera aserrada } \\
\text { Corteza para medic. } \\
\text { Madera aserrada, triplay }\end{array}$ & $\begin{array}{l}\text { Explotado } \\
\text { Localmente explot. } \\
\text { Explotado } \\
\text { Localmente explot. } \\
\text { Explotado } \\
\text { Explotado } \\
\text { Explotado } \\
\text { Localmente explot. } \\
\text { Localmente explot. } \\
\text { Localmente explot. } \\
\text { Localmente explot. } \\
\text { Localmente explot. } \\
\\
\text { Localmente explot. } \\
\text { Localmente explot. } \\
\text { Explotado } \\
\text { Fuertemente explot. } \\
\text { Localmente explot. }\end{array}$ \\
\hline
\end{tabular}

Tabla 3. Datos geográficas y demográficas de los departamentos tropicales bajos de Loreto, Ucayali, y Madre de Dios, en la República del perú. Basado en el INET (1997).

\begin{tabular}{|c|c|c|c|c|c|c|c|c|}
\hline \multirow[t]{2}{*}{ Departamento } & \multirow[t]{2}{*}{$\begin{array}{c}\text { Superficie } \\
\left(\mathbf{k m}^{2}\right)\end{array}$} & \multicolumn{3}{|c|}{ Población } & \multirow{2}{*}{$\begin{array}{c}\text { Población } \\
\text { rural } \\
(\%) \\
1993\end{array}$} & \multirow{2}{*}{$\begin{array}{c}\text { Densidad } \\
\text { Poblacional } \\
\left(\text { hab } / \mathbf{k m}^{2}\right) \\
1993\end{array}$} & \multicolumn{2}{|c|}{$\begin{array}{c}\text { Crecimiento } \\
\text { Poblacional (\%) }\end{array}$} \\
\hline & & 1972 & 1981 & 1993 & & & $\begin{array}{c}1972- \\
1981\end{array}$ & $\begin{array}{c}1981- \\
1993\end{array}$ \\
\hline LORETO & 368852 & 409772 & 516371 & 736161 & 42 & 2,0 & 2,6 & 3,0 \\
\hline UCAYALI & 102411 & 130030 & 178135 & 331824 & 35 & 3,2 & 3,5 & 5,3 \\
\hline MADRE DE DIOS & 85183 & 25154 & 35788 & 69854 & 43 & 0,8 & 3,9 & 5,7 \\
\hline & 556446 & 564956 & 730294 & 1137839 & - & - & - & - \\
\hline
\end{tabular}


Tabla 4. Clave de las formaciones de vegetación forestal de la 1lanura aluvial en la region baja del río Ucayali y río Marañón.

A. Vegetación Leñosa

B. Buen drenaje

C. Bosque mixto ribereño, inundado con agua blanca mixta (cinturón meántrico)

D. Anualmente inundado 0-1 mes

- Etapa de sucesión tardía (lejos del río)

- Etapa de sucesión temprana (cerca del río)

D. Anualmente inundado 1-3 mes

- Etapa de sucesión tardía (lejos del río)

- Etapa de sucesión temprana (cerca del río)

D. Anualmente inundado 3-6 mes

- Etapa de sucesión tardía (lejos del río)

- Etapa de sucesión temprana (cerca del río)

C. Bosque mixto no ribereño, inundado con agua mixta a negra (cuenca de inundación)

- Inundado anualmente 0-1 mes

- Inundado anualmente 1-3 mes

- Inundado anualmente 3-6 mes

B. Drenaje pobre a permanentemente inundado

- Bosque de latifoliadas mixto de lugares ribereños (cinturón meántrico)

- Bosque de latifoliadas mixto de lugares no ribereños (cuenca de inundación)

- Árbol dominante Pseudobombax munguba

- Palmera dominante Mauritia flexuosa

A. Vegetación no leñosa

- Permanente vegetación pantanosa y flotante

- Al menos periódicamente con aguas abiertas

- Vegetación de maleza anual en lugares ribereños arcillosos

- Vegetación de maleza anual en lugares ribereños arenosos

1. Restinga alta

2. Restinga temprana

3. Restinga baja

4. Restinga temprana

5. Tahuampa

6. Tahuampa temprana

7. Restinga alta de cuenca de inundación

8. Restinga baja de cuenca de inundación

9. Tahuampa de cuenca de inundación

10. Bajial

11. Bajial de cuenca de inundación

12. Pungal

13. Aguajal

14. Pantanal

15. Lago y río

16. Barrial

17. Playa 
Tabla 5. Un ejemplo del desarrollo demográfico en un distrito municipal del Perú (Jenaro Herrera) localizado en la zona baja del río Ucayali. Los niveles de población de los caseríos son estimadas de 1995.

Las indicaciones de tierra alta significa que la gente también utiliza la tierra firme para propósitos de subsistencia. Los años de fundación que aparecen marcados con asteriscos son dudosos.

\begin{tabular}{|l|c|c|c|l|}
\hline \multicolumn{1}{|c|}{ Comunidad } & $\begin{array}{c}\text { Tamaño de } \\
\text { la población }\end{array}$ & $\begin{array}{c}\text { Tierra } \\
\text { alta }\end{array}$ & $\begin{array}{c}\text { Año de } \\
\text { fundación }\end{array}$ & \multicolumn{1}{|c|}{ Fundadores } \\
\hline Casa grande & 280 & No & $1930^{*}$ & Indios Cocama cercano a Iricahua \\
Cedro Isla & 300 & No & 1904 & Del departamento de San Martín \\
Florida & 150 & No & 1990 & De Tamshiyacu cerca Iquitos y desde Pucallpa \\
Iricahua & 120 & No & 1905 & Indios Cocamas del canal de Puinahua \\
Jenaro Herrera & 2000 & $\mathrm{Si}$ & 1944 & Habitantes de cuatro comunidades cercanas \\
Nuevo Aucayacu & 200 & $\mathrm{Si}$ & 1982 & Evangelistas del departamento de San Martín \\
Nuevo York & 100 & No & 1993 & Evangelistas cercanos a Cedro Isla y P. Giner \\
Nuevo San Juan & 200 & No & 1989 & De Tamshiyacu cerca a Iquitos \\
Once de Agosto & 150 & No & 1990 & Adyacentes de Yanallpa \\
Padre Giner & 100 & No & $1920^{*}$ & Adyacentes de Cedro Isla \\
Progreso & 250 & No & 1942 & No hay información \\
Pumacahua & 150 & Si & $1930^{*}$ & No hay información \\
Yanallpa & 400 & No & 1911 & Del río Amazonas y del departamento San Martín \\
\hline
\end{tabular}


BOSQUE DE LA LLANURA ALUVIAL DEL PERÚ: ECOSISTEMAS,

굴

की

菑

뎡

in

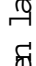

'્4

88

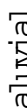

.

(0)

总 8

도

8 ' 8

范

营

응

8 .

б

है

एव

छ

का

.

굴

夏

प्र

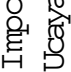

6
$\frac{0}{70}$
$0-1$

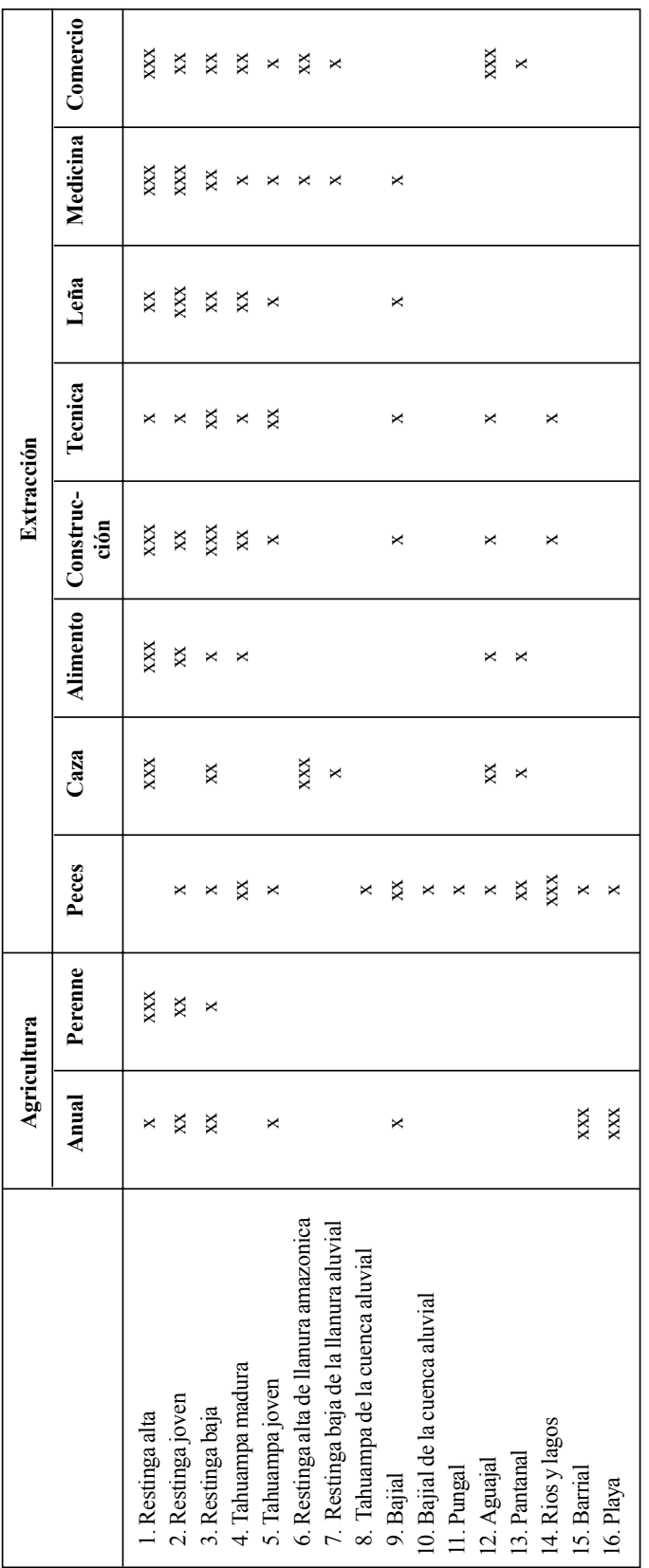


Tabla 7. Extracción y uso de la madera comercial en 1996, en los departamentos de Loreto y Ucayali. Las cifras son volúmenes $\left(\mathrm{m}^{3}\right)$ de madera extraída de tierra fime y llanura aluvial, sólo de llanura aluvial, y según las especies que crecen en la llanura aluvial. Las cifras de la extracción total de especies de la llanura aluvial incluyen volúmenes reales extraídos de pobladores de tierra firme, ya que algunas de las especies seleccionadas crecen en ambas zonas. La producción de triplay y parquet en el departamento de Ucayali no se especificó en especies. Datos sin publicar del Banco Central de Reserva del Perú, Sucursal Iquitos, basado en informes económicos del Ministerio de Agricultura.

\begin{tabular}{|c|c|c|c|c|c|}
\hline & \multicolumn{3}{|c|}{ Loreto } & \multicolumn{2}{|c|}{ Ucayali } \\
\hline & $\begin{array}{c}\text { Madera en } \\
\text { trozas }\end{array}$ & $\begin{array}{l}\text { Madera } \\
\text { aserrada }\end{array}$ & $\begin{array}{c}\text { Triplay } \\
\text { y chapas } \\
\text { decorativas }\end{array}$ & $\begin{array}{l}\text { Madera } \\
\text { aserrada }\end{array}$ & $\begin{array}{l}\text { Triplay y } \\
\text { parquet }\end{array}$ \\
\hline Total & 188930 & 71171 & 25807 & 209976 & 45066 \\
\hline Total especies de la llanura aluvial & 173183 & 66508 & 25807 & 123447 & - \\
\hline Bolaina (Guazuma spp.) & 37 & 47 & - & 473 & - \\
\hline Caoba (Swietenia macrophylla) & 31868 & 22861 & - & 14654 & - \\
\hline Capinuri (Maquira coricea) & 5031 & - & 1053 & - & - \\
\hline Capirona (Calycophyllum spruceanum) & 560 & - & - & 1428 & - \\
\hline Carahuasca (Annonaceae) & - & - & _ & 7 & _ \\
\hline Catahua (Hura crepitans) & 3191 & 1001 & - & 26719 & - \\
\hline Cedro (Cedrela odorata) & 27154 & 17079 & - & 25670 & - \\
\hline Copaiba (Copaifera paupera) & 8295 & 777 & - & 19686 & - \\
\hline Cumala (Virola spp.) & 27884 & 21425 & - & 17247 & - \\
\hline Huayruro (Ormosia spp.) & 120 & - & - & - & - \\
\hline Huimba (Ceiba samauma) & - & - & - & 177 & - \\
\hline Lagarto caspi (Calophyllum brasiliense) & 4353 & 2397 & - & 1182 & - \\
\hline Lupuna (Ceiba pentandra) & 60323 & 49 & 24754 & 3426 & - \\
\hline Mari mari (Leguminosae) & 338 & 50 & - & - & - \\
\hline Moena (Lauraceae) & 3031 & 697 & - & 9999 & - \\
\hline Pashaco (Leguminosae) & 437 & 55 & - & 1794 & - \\
\hline Pumaquiro (Aspidosperma spp.) & 493 & 69 & - & - & - \\
\hline Quinilla (Sapotaceae) & 68 & 1 & - & 333 & - \\
\hline Requia (Sapotaceae) & - & - & - & 266 & - \\
\hline Ubos (Spondias mombim) & - & - & - & 105 & - \\
\hline Yacushapana (Terminalia oblonga) & - & - & - & 281 & - \\
\hline
\end{tabular}


Figura 1. Ell clima de Jenaro Herrera, registrado en campo abierto del Centro de Investigaciones Jenaro Herrera. Todas las figuras están promediadas por mes, sus valores están calculados en base a mediciones diarias.

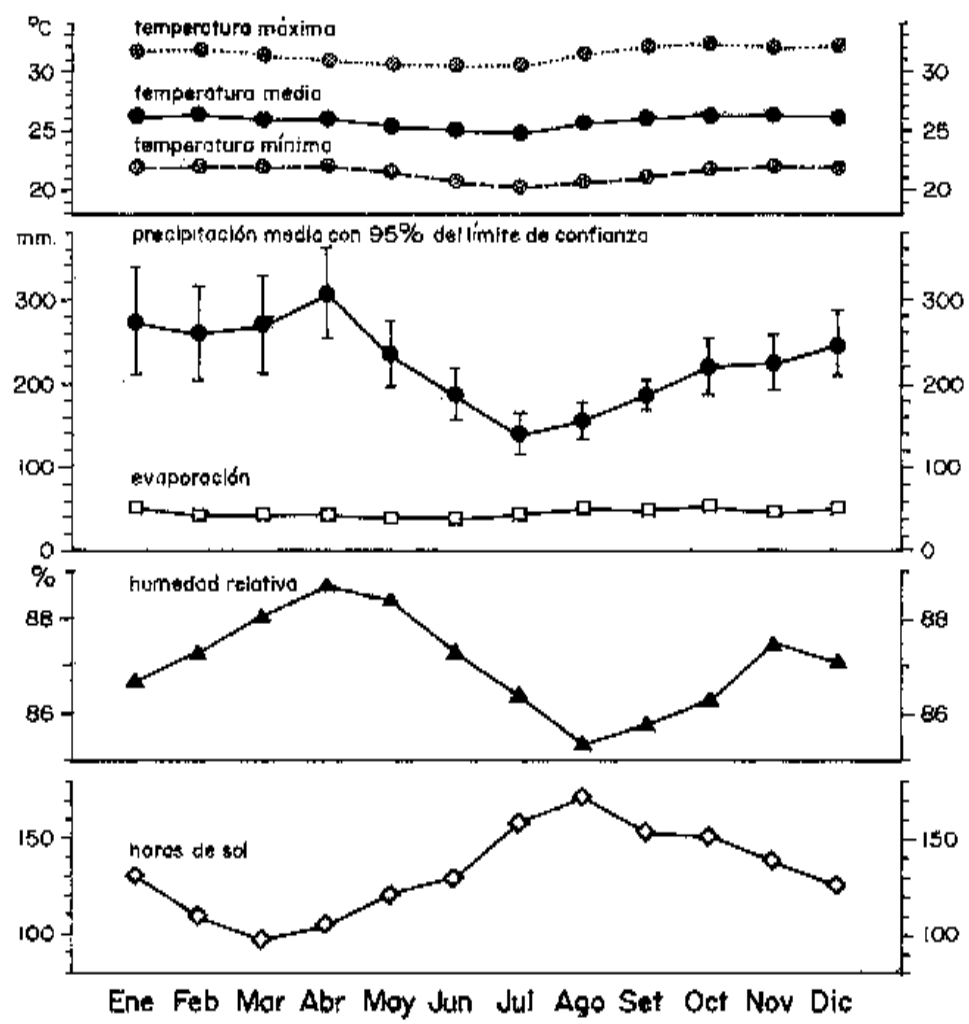



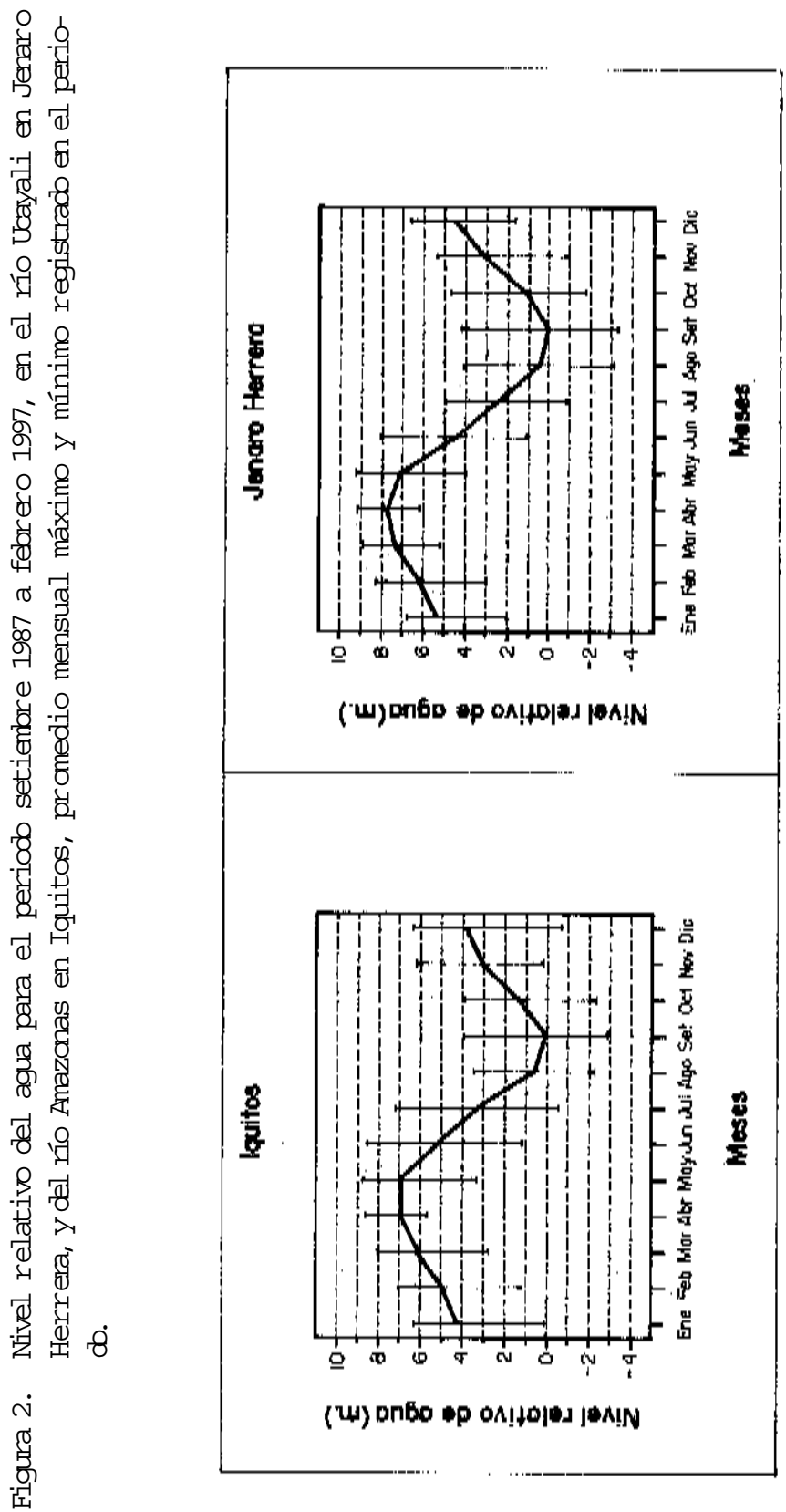

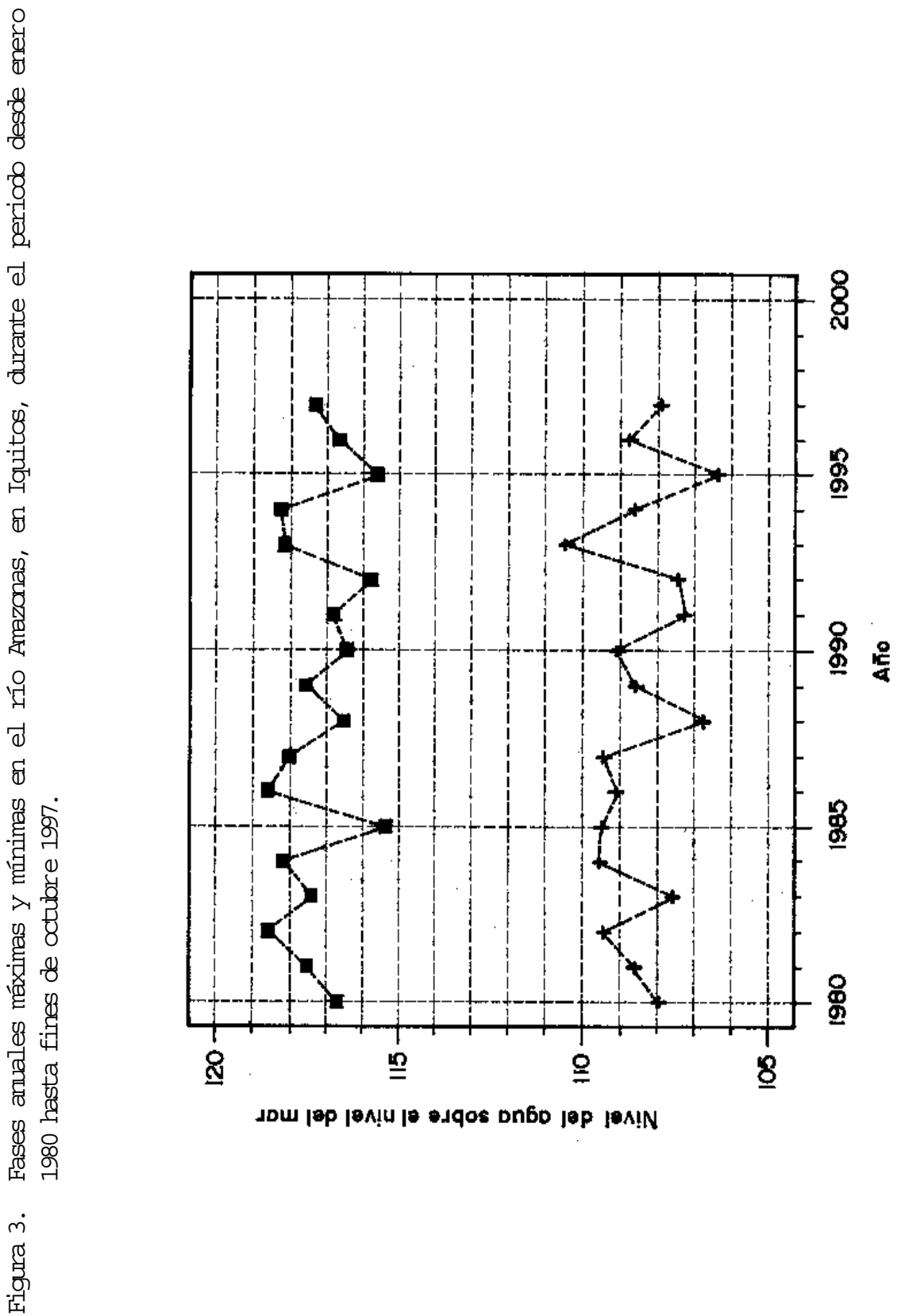